\title{
Composição florística de angiospermas no carste do Alto São Francisco, Minas Gerais, Brasil ${ }^{1}$
}

\author{
Floristic composition of angiosperms in the karst of upper São Francisco river, \\ Minas Gerais state, Brazil
}

\author{
Pablo Hendrigo Alves de Melo ${ }^{2,6}$, Julio Antonio Lombardi ${ }^{3}$, Alexandre Salino ${ }^{4}$ \& Douglas Antônio de Carvalho ${ }^{5}$
}

\begin{abstract}
Resumo
A ocorrência de rochas carbonáticas delimita um tipo particular de relevo, o carste, o qual, pela geomorfologia e hidrologia específicas, difere das paisagens dominantes. Na região cárstica do Alto São Francisco, conhecida como "Mata de Pains", encontram-se exemplos marcantes de relevos cársticos ocorrentes em Minas Gerais. No cárste, a cobertura vegetal é composta por mosaico fitofisionômico apresentando áreas florestadas e áreas abertas, com notável diversidade florística. O objetivo deste estudo foi realizar o levantamento das espécies de angiospermas em áreas com afloramentos de rocha carbonática na Região Cárstica do Alto São Francisco, bem como verificar a relação florística da região estudada com a flora dos domínios fitogeográficos brasileiros. Para o levantamento, no período de 2002 a 2006, foram realizadas expedições mensais para coletas de material botânico fértil concentradas em áreas relacionadas a rochas carbonáticas. O material coletado totalizou 1.512 exsicatas e foi incorporado ao acervo do herbário BHCB. Foram encontradas 456 espécies de angiospermas, distribuídas em 299 gêneros e 77 famílias. O hábito herbáceo foi o melhor representado, com 161 espécies, seguido dos hábitos arbustivo e arbóreo (111 espécies cada), escandente (73 espécies). A flora encontrada na Mata de Pains apresenta influências, em ordem de importância, dos domínios fitogeográficos Mata Atlântica, Cerrado, Amazônia, Caatinga, Pantanal e Pampa. Inventários florísticos em regiões cársticas nos diferentes domínios fitogeográficos podem, em conjunto, fornecer informações importantes no entendimento histórico da vegetação neotropical.
\end{abstract}

Palavras-chave: flora, domínios fitogeográficos, Mata de Pains, calcário, dolomito.

\begin{abstract}
The occurence of carbonatic rocks defines a typical type of relief, called karst, that, by its geomorphology and hydrology, usually differs from the surrounding landscape. In the upper São Francisco river basin, some striking remnants of vegetation associated to karst can be found, which are locally knwon as "Mata de Pains". In this region, a mosaic of different physiognomies, including forests and open areas, which present noteworthy plant diversity, composes the vegetation. The aim of this study is to provide an inventory of angiosperm species in areas of carbonatic rocks outcrops in the upper São Francisco river basin karst region, as well as analyze the floristic relationship of the study area with different Brazilian phytogeographic domains. Fieldwork was performed during the period of 2002 a 2006, when collections of fertile speciemns were done in areas associated to carbonatic rock outcrops. During the study, 1512 exsicates were incorporated to BHCB herbarium collection. A total of 456 angiosperm species were inventoried, distributed in 299 genera and 77 families. Herbaceous habit was better represented, with 161 species, followed by shrubs and trees (111 species each) and lianas (73 species). The flora of the study area presents more influence of the Mata Atlântica domain (Atlantic Rain Forest), followed by, in order of importance, Cerrado, Amazonia, Caatinga, Pantanal and Pampa. Floristic surveys in different karst regions inserted in different phytogeographic domains may, together, provide useful information in understanding the phytogeografic history of Neotropical vegetation. Key words: flora, phytogeographic domains, Mata de Pains, limestone outcrops, dolomite.
\end{abstract}

Este artigo possui material adicional em sua versão eletrônica.

\footnotetext{
${ }^{1}$ Parte da dissertação de Mestrado do primeiro autor.

${ }^{2}$ UFLA - Universidade Federal de Lavras, Depto. Biologia, C.P. 3037, 37200-000, Lavras, MG.

${ }^{3}$ UNESP - Universidade Estadual Paulista Júlio de Mesquita Filho, Inst. Biociências, Depto. Botânica, Av. 24 A 1515, Bala Vista, 13506-900, Rio Claro, SP.

${ }^{4}$ UFMG - Universidade Federal de Minas Gerais, Inst. Ciências Biológicas, Depto. Botânica. Av. Antônio Carlos 6627, Pampulha, 31270-901, Belo Horizonte, MG.

${ }^{5}$ Autor para correspondência: pablopains@yahoo.com.br
} 


\section{Introdução}

Fatores de exceção local, de ordem litológica, hidrológica, topográfica e paleobotânica permitem a existência de "ilhas" de vegetação dentro das áreas núcleo dos diferentes domínios morfoclimáticos e geobotânicos (Ab’Sáber 2005).

Em qualquer domínio morfoclimático, a ocorrência de rochas carbonáticas delimita um tipo particular de relevo, o carste, que perfaz quase $10 \%$ da superfície do território nacional, o qual, pela geomorfologia e hidrologia específica, difere das paisagens dominantes. A presença de afloramentos de rocha carbonática proporciona a formação de paisagens típicas como dolinas, cavernas, maciços residuais, lapiás, paredões, fendas e diáclases (Pilo 2000; Kohler 2002).

A cobertura vegetal também caracteriza a paisagem cárstica, composta por mosaico fitofisionômico apresentando áreas florestadas e áreas abertas. Warming (1973) sugeriu estar na profundidade do solo, e na conseqüente capacidade de retenção d'água, o principal fator de distinção ambiental entre a formação sempre florestal na base das rochas e a capoeira seca muito aberta que cresce por cima das rochas. Cada feição cárstica apresenta uma série de características ambientais distintivas, como a disponibilidade e tipo de substrato, diferentes condições de umidade e de luminosidade, que somadas, constituem micro-habitats específicos, que por sua vez, suportam, cada qual, diferentes tipos de vegetação, tornando-o, no conjunto, rico e variado, com espécies, muitas vezes restritas a estes ambientes.

A região cárstica do Alto São Francisco, região conhecida como "Mata de Pains" (MelloBarreto 1942; Barbosa 1961), apresenta exemplos marcantes dos relevos cársticos ocorrentes nas Folhas SF.23/24 Rio de Janeiro/Vitória (Barbosa 1961; RADAMBRASIL 1983). Mello-Barreto (1942) destacou a região da Mata de Pains pela presença de matas pujantes relacionadas aos terrenos calcários. Eschwege (1979), que em 1800 percorreu a região à procura de reservas de salitre, destacou o contraste entre as formações florestais existentes na base dos afloramentos e áreas abertas, existentes nos locais de rocha exposta.

São raros os trabalhos de levantamento florístico realizados em áreas cársticas, por meio da coleta de material botânico fértil durante vários anos, e que considere as distintas fitofisionomias relacionadas ao relevo, bem como todas as formas de vida de angiospermas (Warming 1973; Pedralli 1997; Lombardi et al. 2005), contudo levantamentos fitossociológicos, como os de Silva \& Scariot (2003, 2004 a,b) e Felfili et al. (2007), Santos et al. (2007) e os de Meguro et al. (2007) tem revelado a riqueza e a diversidade da comunidade arbórea de florestas estacionais decíduas nos estados Goiás e Minas Gerais.

Este estudo teve como objetivo realizar o levantamento florístico de áreas com afloramentos de rocha carbonática na Mata de Pains, Região Cárstica do Alto São Francisco, bem como verificar a relação florística da região estudada com a flora dos domínios fitogeográficos da Mata Atlântica, Cerrado e Caatinga.

\section{Material e Métodos}

Localização e caracterização ambiental da área de estudo

A Mata de Pains está localizada no sudoeste do estado de Minas Gerais, no alto curso do Rio São Francisco, englobando a totalidade dos municípios de Pains e Doresópolis, além de partes dos municípios de Arcos, Córrego Fundo, Formiga, Iguatama, Pimenta e Piumhí (Fig.1).

Considerando os dados da estação meteorológica mais próxima da área de estudo, Bambuí, o clima, pela classificação de Köppen, é do tipo Cwa, temperado brando com verão quente e úmido e inverno seco. A temperatura média anual é de $20,7^{\circ} \mathrm{C}$, sendo julho o mês mais frio, com temperatura média de $16,3^{\circ} \mathrm{C}$. A precipitação média anual local é de $1.344 \mathrm{~mm}$.

A geologia regional é caracterizada pela ocorrência das rochas carbonáticas e silto-argilosas pertencentes ao Grupo Bambuí, Proterozóico Superior, no limite sudoeste da porção sul do Cráton do São Francisco. Apresenta um mosaico de afloramentos calcários e filitos sucessivos, permitindo a ocorrência de zonas cársticas isoladas em meio a rochas não carbonáticas. Destacam-se na área três domínios cársticos, separados por ocorrências de filitos: o de Pains, de Arcos e o de Doresópolis, que, apesar de em certos casos não terem limites muito precisos, são individualizados pelas concentrações de formas cársticas. As águas superficiais distribuem-se por rede hidrográfica tipicamente pouco densa, favorecida pela infiltração direta nas fissuras da rocha calcária e pelas feições de absorção cársticas, sumidouros, simas, etc. Destacam-se os seguintes cursos d'água principais: Rio São Francisco, que compreende o nível de base regional, Rio São Miguel e Ribeirão dos Patos (Menegasse et al. 2002). O Rio São Miguel constitui um importante tributário da margem direita do Rio São Francisco. O seu alto curso apresenta uma 

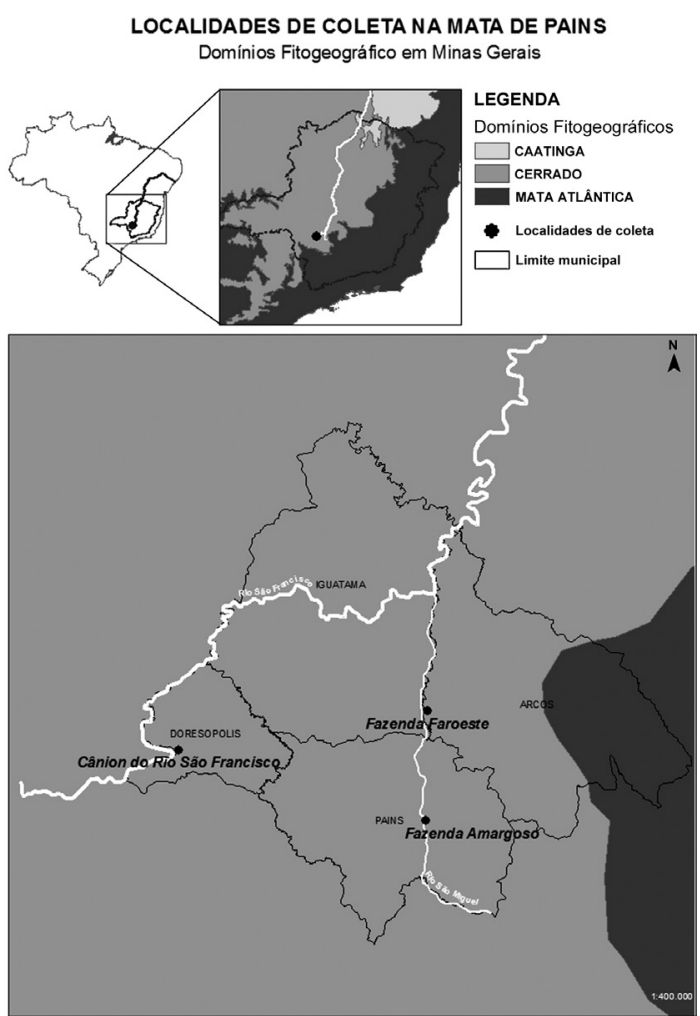

Figura 1 - Localização das áreas onde se realizou a coleta de material botânico. 1. Fazenda Faroeste nos municípios de Arcos e Iguatama; 2. Fazenda Amargoso no município de Pains; 3. Cânion calcário do Alto São Francisco no município de Doresópolis.

Figure 1 - Location of the areas where collection of botanical material were done. 1. Faroeste Farm, in Arcos and Iguatama municipalities; 2. Amargoso Farm, in Pains municipality; 3. Karst canyon of upper São Francisco river, in Doresópolis municipality

grande concentração de feições cársticas, enquanto o baixo curso ocorre em uma planície alagada.

De 2002 a 2005 foram realizadas expedições mensais em duas fazendas da região, ao longo da bacia do Rio São Miguel, Fazenda Amargoso $\left(20^{\circ} 23^{\prime} 14\right.$ 'S e $\left.45^{\circ} 38^{\prime} 59^{\prime \prime} \mathrm{W}\right)$, a montante, no município de Pains, e Fazenda Faroeste $\left(20^{\circ} 15^{\prime} 05^{\prime}\right.$ 'S e 4539'46"W), a jusante, estendendo-se pelos municípios de Arcos e Iguatama. A Fazenda Amargoso possui aproximadamente 50 ha cobertos por fragmentos de Floresta Estacional Decidual e Semidecidual na base e sobre os afloramentos rochosos que ocorrem nas encostas das colinas e se conectam ao Parque Municipal Dona Ziza, localizado na periferia da cidade de Pains. A Fazenda Faroeste representa uma das áreas naturais mais importantes e representativas da região, com aproximadamente 1000 ha cobertos por Floresta
Estacional Decidual e Semidecidual relacionadas aos afloramentos rochosos que guardam 27 sítios arqueológicos já catalogados. Os maciços aflorados margeiam o Rio São Miguel formando um vale raso e largo que alaga no final da estação chuvosa. No ano 2006 realizaram-se também expedições ao município de Doresópolis perfazendo as áreas do cânion calcário do Alto São Francisco (20 $18^{\circ} 25^{\prime \prime}$ S e 455' $\left.08^{\prime \prime} \mathrm{W}\right)$.

O levantamento florístico contemplou todos os hábitos de angiospermas. Em cada localidade amostrada, por meio de caminhadas aleatórias, percorreram-se os diversos ambientes como bordas, topos e interior de maciços residuais, lajedos de calcário, bordas de cânion e paredes marginais a cursos d'água, vertentes rochosas afloradas em pequenos morros e serras, interior de clarabóias e dolinas. Os espécimes foram coletados em estado fértil e herborizados conforme as técnicas padrão para estudos desse tipo (Fidalgo \& Bononi 1984), e, quando existentes, duplicatas foram enviadas a especialistas para identificação. Outros espécimes foram identificados pelos autores por comparação ou por consulta à literatura especializada. Todas as exsicatas estão depositadas no Herbário do Departamento de Botânica do Instituto de Ciências Biológicas da Universidade Federal de Minas Gerais (BHCB).

Adotou-se o sistema de classificação apresentado por APG II (Souza \& Lorenzi 2008). Os nomes das espécies foram atualizados quanto à sinonímia consultando-se o sítio do Taxonomic Name Resolution Service $(<\mathrm{http}: / / \mathrm{tnrs}$.iplantcollaborative. org/>). O hábito dos espécimes coletados estão de acordo com Vidal \& Vidal (2000).

Foram usados os levantamentos de Forzza et al. (2011), Stehmann et al. (2009), Mendonça et al. (1998) e Queiroz et al. (2006), para verificar a relação florística da região estudada com a flora dos domínios fitogeográficos no Brasil. Dados de ocorrência por formação vegetacional nos domínios Mata Atlântica, Cerrado e Caatinga foram obtidos de Stehmann et al. (2009), Mendonça et al. (1998) e Queiroz et al. (2006), respectivamente.

\section{Resultados e Discussão}

De modo geral constatou-se predomínio de Floresta Estacional Semidecidual na base e entre os afloramentos. Sobre estes, em locais com presença do epicarste, ou seja, locais onde há solo recobrindo a rocha ou preenchendo seus interstícios, ocorre a Floresta Estacional Decidual. Nos locais mais 
erodidos, como nos campos de lapiás, ocorre uma terceira fitofisionomia, saxícola, mais aberta, particular das partes mais dissecadas do relevo cárstico, aqui chamada de formação aberta do carste (Fig. 2).

Foram encontradas 456 espécies de angiospermas, distribuídas em 299 gêneros e 77 famílias. A listagem de espécies é apresentada na Tabela 1. As famílias com maior número de espécies foram: Fabaceae (37), Orchidaceae (28), Asteraceae (25), Poaceae e Malvaceae (24), Euphorbiaceae (23), Piperaceae (19), Apocynaceae, Bromeliaceae e Rubiaceae (14 cada), Solanaceae (13), Bignoniaceae (11) e Meliaceae (10).

$\mathrm{O}$ número de espécies amostradas excede àqueles relatados em trabalhos florísticos e/ou fitossociológicos realizados em outras áreas de afloramentos calcários no estado de Minas Gerais (Azevedo 1966; Pedersoli \& Martins 1972; Brandão \& Magalhães 1991; Pedralli 1997; Lombardi et al. 2005; Meguro et al. 2007; Santos et al. 2007) e em
Goiás (Silva \& Scariot 2003, 2004a, b; Felfili et al. 2007). A alta riqueza de espécies encontrada se deve ao fato de ter-se amostrado, em expedições periódicas durante três anos, todas as formas de vida, nas distintas fitofisionomias relacionadas ao relevo cárstico, bem como seus diferentes micro-habitats. Warming (1973) observou a variação nas condições do relevo como um importante fator no incremento da riqueza de espécies no carste e ressaltou a necessidade de expedições frequentes para a coleta de material botânico fértil nessas regiões.

O hábito herbáceo foi o melhor representado, com 161 espécies, seguido dos hábitos arbustivo e arbóreo (111 espécies cada), escandente (73 espécies). Os gêneros com espécies herbáceas com maior riqueza de espécies foram: Peperomia (13), Tillandsia (6), Begonia e Panicum (4 cada); com espécies arbustivas: Piper (5), Psychotria e Solanum (4 cada); com espécies arbóreas: Trichilia

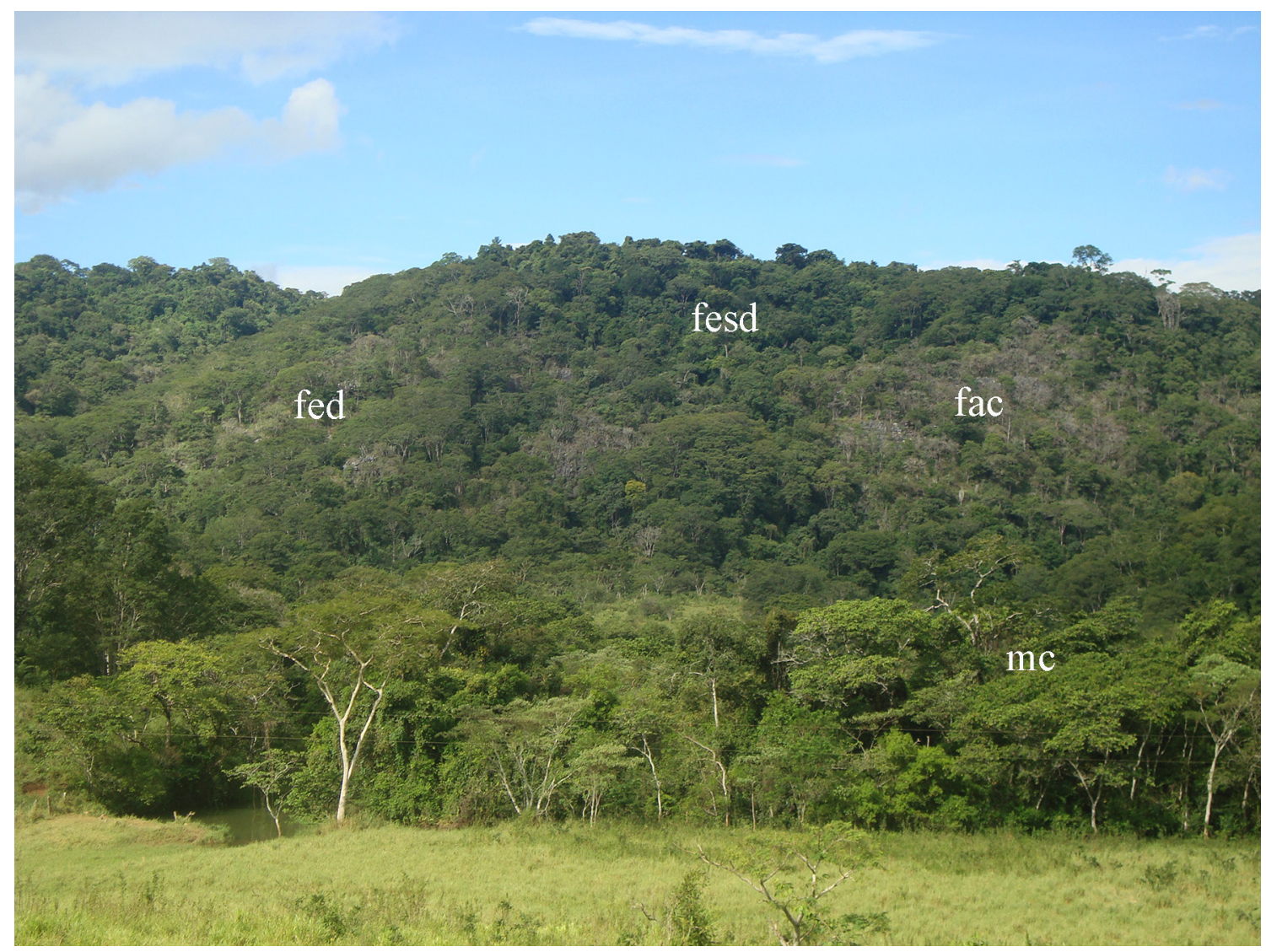

Figura 2-Mosaico fitofisionômico apresentando áreas florestadas e áreas abertas na Fazenda Faroeste em 15.03.2012. fesd=floresta estacional semindecidual, fed=floresta estacional decidual, fac=formação aberta do carste e mc=mata ciliar do rio São Miguel. Figure 2 - Mosaic of different physiognomies, including forests and open areas. Fazenda Faroeste in 03.15.2012. fesd=seasonal semideciduous forest, fed=seasonal deciduous forest, $\mathrm{fac}=\mathrm{open}$ formations of carste and $\mathrm{mc}=$ riparian forest of São Miguel river. 
(5), Nectandra (4), Casearia, Croton, Eugenia, Ficus, Guarea e Lonchocarpus (3 cada); e com espécies escandentes: Cissus (6), Dioscorea (5), Prestonia (4), Forsteronia e Passiflora (3) cada.

Rizzini (1997) destacou relativa pobreza de espécies herbáceas no sub-bosque das formações florestais decíduas sobre afloramentos calcários no estado de Goiás, porém na Mata de Pains, considerando-se as distintas fitofisionomias relacionadas ao relevo cárstico, as espécies herbáceas representaram cerca de um terço da riqueza total de espécies encontradas.

Pedersoli \& Martins (1972) sugerem que o estrato herbáceo sobre afloramentos calcários pode ser dividido em uma formação heliófila que cresce sobre a rocha, e outra ombrófila, mais rica em espécies, em razão de uma espessa camada de húmus. Neste levantamento os locais mais erodidos e dissecados do relevo são caracterizados por espécies como: Loasa rostrata, Gardnerina angustata (Gardner) R.M. King \& H. Rob., Hylocereus setaceus (Salm-Dyck) Ralf Bauer, Pilea microphylla (L.) Liebm., Sinningia warmingii (Hiern) Chautems, Tillandsia polystachia (L.) L., T. streptocarpa Baker e Urera spp., entre outras, Encholirium luxor L.B. Sm. \& Read parece ser uma das espécies mais típicas de áreas com lapiés. Já no sub-bosque das formações florestais ocorrem varias espécies de Marantaceae e espécies dos gêneros Olyra e Dorstenia. Muitas espécies de ervas, principalmente das famílias Araceae, Bromeliaceae, Cactaceae e Orchidaceae, crescem como rupícolas, saxícoloas ou epífitas.

Andrade-Lima (1977) destaca ervas de forma de vida terófita e geófita, como de presença certa, embora inconspícua na maior parte do ano. As coletas realizadas neste levantamento permitiram a redescoberta da terófita, Gardnerina angustata (Warming 1973), e a descoberta de espécie não descrita de novo gênero da família Gesneriaceae, Chautemsia calcicola A.O.Araujo \& V.C.Souza, uma geófita (Araujo et al. 2010). Nos interstícios das paredes e das encostas na base dos afloramentos, ambientes sazonalmente mais úmidos devido ao gotejamento d'água, é comum, somente na estação chuvosa, encontrar-se ervas geófitas como Asterostigma lombardii E.G.Gonç., Begonia sp 1., Peperomia gardneriana Miq., Sinningia conspicua (Seem.) G. Nicholson e $S$. warmingii (Hiern) Chautems.

A flora encontrada na Mata de Pains apresenta influências, em ordem de importância, dos domínios fitogeográficos Mata Atlântica (com 288 espécies comuns), Cerrado (246 espécies) e Caatinga (186 espécies), de acordo com as listagens florísticas de Stehmann et al. (2009), Mendonça et al. (1998) e Queiroz et al. (2006), respectivamente. (Fig. 3a). As Figuras $3 b-d$ evidenciam maior representatividade de espécies de formações florestais nos domínios
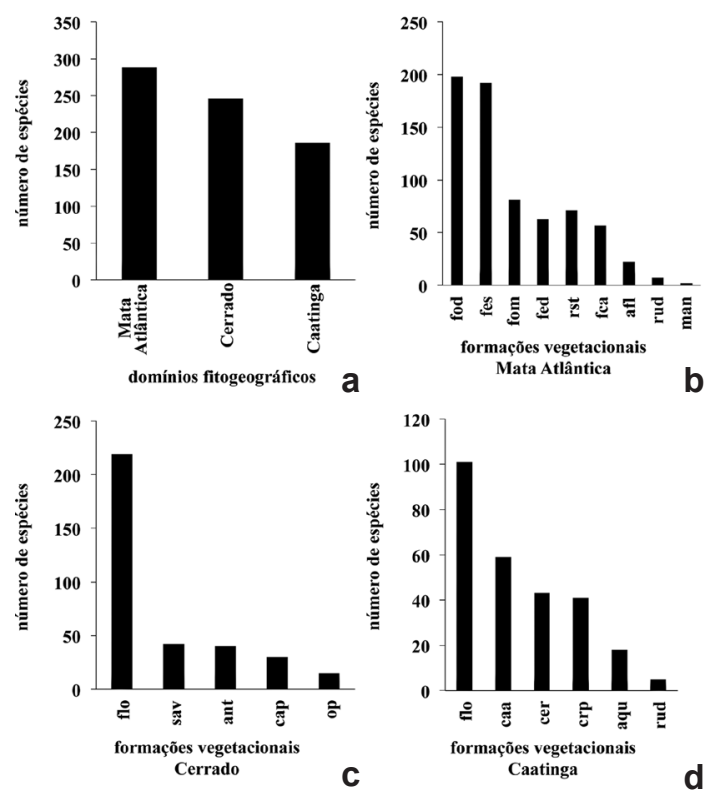

Figura 3 - Riqueza de espécies de angiospermas coletadas na Mata de Pains por a. domínio fitogeográfico; b. formação vegetacional na mata atlântica segundo Stehmann et al. (2009). fod=floresta ombrófila densa, fes=floresta estacional semindecidual, fom=floresta onbrófila mista, fed=floresta estacional decidual, rst=restinga, fca=formações campestres, afl=afloramentos rochosos, rud=ruderal, man=mangue; c. formação vegetacional no cerrado segundo Mendonça et al. (1998). flo=florestal, sav=savânica, ant=antrópica, cam $=$ campestre, $o p=$ outras paisagens; $d$. formação vegetacional na caatinga segundo Queiroz et al. (2006). flo=floresta, caa=caatinga, cer $=$ cerrado, $\mathrm{crp}=$ campo rupestre, $\mathrm{aqu}=$ brejos e ambientes aquáticos, rud-ruderal.

Figure 3 - Angiosperm species richness in the study area distributed by its occurrence in: a. phytogeographic domain; b. phytophysiognomy in the Atlantic Rain Forest, according Stehmann et al. (2009). fod=evergreen dense forest, fes=seasonal semideciduous forest, $\mathrm{rst}=$ coastal sandbanks, $\mathrm{fca}=$ open formations, afl=rock outcrops, rud=antropized areas, man=mangroves; c. phytophysiognomy in the Cerrado, according Mendonça et al. (1998). flo=forests, sav=savanna, ant=antropized areas, cam=open fields, op=other formation; d. phytophysiognomy in the Caatinga, according to Quieroz et al. (2006). flo=forests, caa=caatinga, cer $=$ cerrado, crp $=$ rocky grasslands, aqu $=$ bogs and aquatic environments, rud-antropized areas. 
fitogeográficos da Mata Atlântica, Cerrado e Caatinga. Considerando a listagem de Forzza et al. (2011), verifica-se, em ordem de importância, influência da Mata Atlântica (com 307 espécies em comum), Cerrado (265 espécies), Amazônia (176 espécies), Caatinga (170 espécies), Pantanal (45 espécies) e Pampa (24 espécies) (Fig. 4).

Das espécies registradas para a Mata de Pains, 38 não contam como ocorrentes em Minas Gerais segundo Forzza et al. (2011) (Tab. 1), destacandose: Abutilon fluviatile (Vell.) K.Schum., Cedrela odorata L., Chamaeranthemum gaudichaudii Ness, Cordia tetrandra Aubl., Dorstenia caatingae R.M.Castro, Gardnerina angustata (Gardner) R.M. King \& H. Rob., Lonchocarpus sericeus (Poir.) DC., Manihot grahamii Hook., Notylia hemitricha Barb. Rodr., Pilocarpus pauciflorus A.St.-Hil., Pisonia aculeata L., Romanoa tamnoides (A.Juss.) Radcl.-Sm. e Wissadula macrantha R.E.Fries.

Os resultados demonstram expressiva contribuição de espécies herbáceas na composição florística da área estudada. Geófitas e terófitas assim como a caducifolia do componente arbóreo, estampam o caráter sazonal da vegetação de regiões cársticas. Ressalta-se, portanto, a importância de uma amostragem que considere todas as fitofisionomias e formas de vida presentes para uma melhor compreensão da real riqueza da flora relacionada ao relevo cárstico.

Há mais de dois séculos a atividade de mineração é realizada na região Mata de Pains. Inicialmente retirava-se o solo salitroso de cavernas para produção de pólvora (Eschwege 1979). Atualmente são extraídos os maciços de rocha carbonática para a produção de corretivo de solo para a agricultura, cimento e cal.

Além de grande riqueza florística a Mata de Pains é a região que registra a maior concentração de cavernas do Brasil, com mais de 1.000 cavidades registradas (Ferreira 2009) e 200 sítios arqueológicos conhecidos (Henrique Junior 2006). É de importância biológica extrema para conservação da biodiversidade em Minas Gerais (Drummond et al. 2005). Entretanto, dos seus $1.500 \mathrm{~km}^{2}$, menos de 400 hectares estão efetivamente protegidos por duas unidades de conservação, uma RPPN de 83 ha e uma Estação Ecológica de 304 ha, concentradas na sub-bacia do córrego Candongas, ente os municípios de Pains e Arcos.

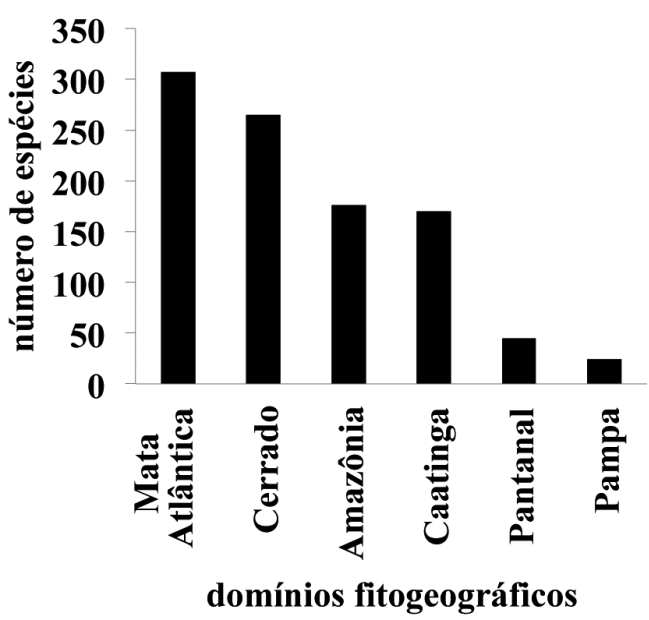

Figura 4 - A ocorrência das espécies de angiospermas coletadas na Mata de Pains por domínio fitogeográfico segundo Forzza et al. (2010).

Figure 4 - Occurrence of the inventoried species in phytogeographic domain according to Forzza et al. (2010).

As grandes dimensões do principal remanescente florestal encontrado na região fazem da Fazenda Faroeste uma área relevante para preservação por meio da criação de unidade de conservação na bacia do rio São Miguel. Grandes áreas de formação aberta relacionada ao relevo cárstico ocorrem na bacia do Ribeirão dos Patos. Nessa bacia destaca-se as regiões do córrego Cavalo e do córrego Barreado, cujos cursos se perdem sob os maciços, em cavernas e sumidouros. E a região do Mato das Frutas onde ocorre grande concentração lagoas cársticas. No contexto regional merece ainda destaque o cânion calcário do Rio São Francisco pela singular beleza cênica.

\section{Agradecimentos}

Os autores agradecem aos especialistas as determinações efetuadas. Aos proprietários de todas as fazendas estudadas, em especial a José Francisco Gonçalves, da fazenda Amargoso e a Tasso Assunção (em memória) e família, da fazenda Faroeste, o longo período de apoio e a confiança. Ao casal Ernani Melo e Elizabete Rodrigues o apoio logístico em campo. Aos pesquisadores Pedro Viana, Felipe Leite e Ernani Júnior o apoio em todas as etapas deste trabalho. Ao $\mathrm{CNPq}-$ Conselho Nacional de Desenvolvimento Científico e Tecnológico, pela bolsa de mestrado do primeiro autor pela bolsa produtividade em pesquisa $n^{\circ}$ 300240/2009-0 ao segundo autor. 


\section{Referências}

Ab'saber, A.N. 2005. Potencialidades paisagísticas brasileiras. In: Ab'saber, A.N. (ed.). Domínios de natureza no Brasil: potencialidades paisagísticas. Ateliê Editorial, São Paulo. 160p.

Andrade-Lima, D. 1977. A flora de área erodidas de Calcário Bambuí, em Bom Jesus da Lapa, Bahia. Revista Brasileira de Biologia 37: 179-194.

Azevedo, L.G. 1966. Tipos eco-fisionômicos da vegetação da região de Januária (MG). Anais da Academia Brasileira de Ciências 38: 39-57.

Barbosa, G.V. 1961. Noticias sobre o Karst na Mata de Pains. Boletim Mineiro de Geografia 2: 3-21.

Brandão, M. \& Magalhães, G.M. 1991. Cobertura vegetal da microrregião Sanfranciscana de Januária. Daphne 1: $19-26$.

Drummond, G.M.; Martins, C.M.; Machado, A.B.M.; Sebaio, F.A. \& Antonini, Y. 2005. Biodiversidade em Minas Gerais: um atlas para sua conservação. Belo Horizonte, Fundação Biodiversitas. 222p.

Eschwege, W.L. von. 1979. Pluto brasiliensis. Ed. Itatiaia, São Paulo. 306p.

Felfili, J.M.; Nascimento, A.R.T.; Fagg, C.W. \& Meirelles, E.M. 2007. Floristic compositoin and structure of a seasonally deciduous forest on limestone outcrops in Central Brazil. Revista Brasileira de Botânica 30: 611-621.

Ferreira, R.L.; Silva, M. S. \& Bernardi, L.F.O. 2009. Contexto Bioespeleológico. In: Drummond, G.M.; Martins, C.S.; Greco, M.B. \& Vieira. F. (orgs.). Biota Minas: diagnóstico do conhecimento sobre a biodiversidade no estado de Minas Gerais. Fundação Biodiversitas, Belo Horizonte. Pp.160-179.

Fidalgo, O. \& Bononi, V.L.R. 1984. Técnicas de coleta, preservação e herborização de material botânico. Instituto de Botânica, São Paulo. 62p.

Forzza, R.C.; Leitman, P.; Walter, B.M.T.; Costa, A.; Pirani, J.R.; Morim, M.P.; Queiroz, L.P.; Martinelli, G.; Peixoto, A.L.; Coelho, M.A.N.; Stehmann, J.R.; Baumgratz, J.F.A.; Lohmann, L.G. \& Hopkins, M. 2010. Angiospermas. In: Forzza, R.C. et al. (eds.). Lista de espécies da flora do Brasil. Jardim Botânico do Rio de Janeiro. Disponível em $<$ http:// floradobrasil.jbrj.gov.br/2010/FB000032>. Acesso em 15 Mar 2012.

Henriques Junior, G. P. 2006. Arqueologia regional da província cárstica do Alto São Francisco: um estudo das tradições ceramistas Una e Sapucaí. Dissertação de Mestrado, Museu de Arqueologia e Etnologia, Universidade de São Paulo, São Paulo. Disponível em <http://www.teses.usp.br/teses/ disponiveis/71/71131/tde-23012007-150503/>. Acesso em 15 Mar 2012.

Kohler, H.C. 2002. A escala na análise geomorfológica. Revista Brasileira de Geomorfologia 3: 11-19.
Lombardi, J.A.; Salino, A. \& Temoni, L.G. 2005. Diversidade florística de plantas vasculares no município de Januária, Minas Gerais, Brasil. Lundiana 6: 3-20.

Meguro, M.; Pirani, J.R.; Mello-Silva, R. \& Cordeiro, I. 2007. Composição florística e estrutura das florestas estacionais decíduas sobre calcário a oeste da Cadeia do Espinhaço, Minas Gerais, Brasil. Boletim de Botânica (USP) 25: 147-171.

Mello-Barreto, H.L. 1942. Regiões fitogeográficas de Minas Gerais. Boletim Geográfico 14: 14-28.

Mendonça, R.C.; Felfili, J.M.; Walter, B.M.T.; Silva Jr., M.C.; Rezende, A.V.; Filgueiras, T.S. \& Nogueira, P.E. 1998. Flora vascular do Cerrado. In: Sano, S.M. \& Almeida, S.P. (eds.). Cerrado: ambiente e flora. EMBRAPA-CPAC, Brasília. Pp. 289-556.

Menegasse, L.N.; Gonçalves, J.M. \& Fantinel, L.M. 2002. Disponibilidades hídricas na Província cárstica de Arcos-Pains-Doresópolis, Alto São Francisco, Minas Gerais, Brasil. Revista Águas Subterrâneas 16: 1-19.

Pedersoli, J.L. \& Martins, J. L. 1972. A vegetação dos afloramentos de calcário. Oréades 5: 27-29.

Pedralli, G. 1997. Florestas secas sobre afloramentos de calcário em Minas Gerais: florística e fisionomia. Bios 5: 81-88.

Piló, L.B. 2000. Geomorfologia cárstica. Revista Brasileira de Geomorfologia 1: 88-102.

Queiroz, L.P.; Conceicao, A. \& Giulietti, A.M. 2006. Nordeste Semi-árido: caracterização geral e lista das fanerógamas. In: Giulietti, A.M. \& Queiroz, L.P. (orgs.). Instituto do Milênio do Semi-árido: diversidade e caracterização das fanerógamas do semi-árido brasileiro. Vol. 1. APNE, Recife. Pp. 15-364.

Radam Brasil. 1983. Levantamento de recursos naturais. Vol. 32. Rio de Janeiro: MME/SG. Folhas Sf 23/24 - Rio de Janeiro/Vitória. 775p.

Rizzini, C.T. 1997. Tratado de fitogeografia do Brasil. 2a ed. Âmbito Cultural, Rio de Janeiro. 747p.

Santos, R.M.; Vieira, F.A.; Fagundes, M.; Nunes, Y.R.F. \& Gusmão, E. 2007. Riqueza e similaridade florística de 8 remanescentes florestais no norte de Minas Gerais, Brasil. Revista Árvore 31: 135-144.

Silva, L.A. \& Scariot, A. 2003. Composição florística e estrutura da comunidade arbórea em uma floresta estacional decidual em afloramento calcário (fazenda São José, São Domingos, GO, bacia do rio Paraná). Acta Botanica Brasilica 17: 305-313.

Silva, L.A. \& Scariot, A. 2004a. Comunidade arbórea de uma floresta estacional decídua sobre afloramento calcário na bacia do rio Paraná. Revista Árvore 28: 61-67.

Silva, L.A. \& Scariot, A. 2004b. Composição e estrutura da comunidade arbórea de uma floresta estacional decidual sobre afloramento calcário no Brasil central. Revista Árvore 28: 69-75. 
Souza, V.C. \& Lorenzi, H. 2008. Botânica sistemática: guia ilustrado para identificação das famílias de fanerógamas nativas e exóticas no Brasil, baseado na APG II. $2^{\mathrm{a}}$ ed. Instituto Plantarum, Nova Odessa. 704p.

Stehmann, J.R.; Forzza, R.; Salino, A.; Sobral, M; Costa, D.P. \& Kamino, L.H.Y. (org.). 2009. Plantas da Floresta Atlântica. Jardim Botânico do Rio de Janeiro, Rio de Janeiro. 516p.
Vidal, W.N. \& Vidal, M.R.R. 2000. Botânica organografia; quadros sinóticos ilustrados de fanerógamos. $4^{\mathrm{a}}$ ed. Ed. UFV, Viçosa. 124p.

Warming, E. 1973. Lagoa Santa: contribuição para a geographia phytobotanica. EDUSP/Livraria Itatiaia, Belo Horizonte. 362p.

Taxonomic Name Resolution Service. Disponível em $<$ http://tnrs.iplantcollaborative.org/>. Acesso em 7 Jul 2011. 


\title{
Composição florística de angiospermas no carste do Alto São Francisco, Minas Gerais, Brasil
}

\author{
Floristic composition of angiosperms in the karst of upper São Francisco river, \\ Minas Gerais state, Brazil
}

Pablo Hendrigo Alves de Melo, Julio Antonio Lombardi, Alexandre Salino \& Douglas Antônio de Carvalho

\begin{abstract}
Apêndice - Espécies de angiospermas coletadas em ambientes de afloramentos de rocha carbonática na região cárstica do Alto São Francisco, municípios de Arcos, Doresópolis, Iguatama e Pains, Minas Gerais, cujas exsicatas foram incorporadas nos Herbário BHCB (números de coleta P.H.A.Melo). Hábito: arb = arbusto, árv $=$ árvore, erv $=$ erva, trep $=$ trepadeira; CA1 = Ocorre na caatinga segundo Queiroz et al. (2006), CE2 = ocorre no cerrado segundo Mendonça et al. (1998), MA3 = ocorre na Mata Atlântica segundo Stehmann et al. (2009), AM4 = Ocorre na Amazônia segundo Forzza et al. (2010), CA4 = Ocorre na Caatinga segundo Forzza et al. (2010), CE4 = Ocorre no Cerrado segundo Forzza et al. (2010), MA4 = Ocorre na Mata atlântica segundo Forzza et al. (2010), PP4 = Ocorre nos Pampas segundo Forzza et al. (2011), PT4 = Ocorre no o Pantanal segundo Forzza et al. (2010).

Appendix - Angiosperm species collected in carbonatic rock outcrops in the karst region of the upper São Francisco river, municipalities of Arcos, Doresópolis, Iguatama and Pains, Minas Gerais stated. Specimens deposited in BHCB herbarium (collector

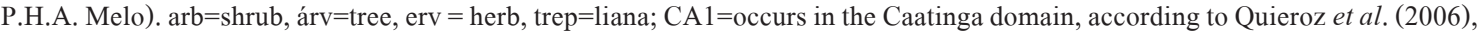
CE2 =occurs in the Cerrado domain, according Mendonça et al. (1998), MA3=occurs in the Mata Atlântica (Atlantic Rain Forest), according Stehmann et al. (2009), AM4=occurs in the Amazônia (Amazon Rain Forest) according to Forzza et al. (2010), CE4=0ccurs in the Cerrado according to Forzza et al. (2010), PP4=occurs in the Pampas according to Forzza et al. (2010), PT4=occurs in the Pantanal according to Forzza et al. (2010).
\end{abstract}

\begin{tabular}{|c|c|c|c|c|c|c|c|c|c|c|c|}
\hline FAMÍLIA/ESPÉCIE & $\begin{array}{l}\text { Melo, } \\
\text { P.H.A }\end{array}$ & Hábito & CA1 & CE2 & MA3 & AM4 & CA4 & CE4 & MA4 & PP4 & PT4 \\
\hline \multicolumn{12}{|l|}{ ACANTHACEAE } \\
\hline Aphelandra longiflora (Lind1.) Profice & 664 & arb & & & & $\mathrm{x}$ & & $\mathrm{x}$ & $\mathrm{x}$ & & \\
\hline Aphelandra schottiana (Nees) Profice & 661 & arb & $\mathrm{x}$ & $\mathrm{x}$ & $\mathrm{x}$ & & & & $\mathrm{x}$ & & \\
\hline Chamaeranthemum beyrichii Nees & 1247 & arb & & & $\mathrm{x}$ & & & & $\mathrm{x}$ & & \\
\hline Chamaeranthemum gaudichaudii Nees & 70 & arb & & & $\mathrm{x}$ & & & & $\mathrm{x}$ & & \\
\hline Justicia cf. brasiliana Roth & 434 & arb & & & $\mathrm{x}$ & & & & $\mathrm{x}$ & & \\
\hline Justicia cf. lythroides (Nees) V.A.W. Graham & 640 & arb & & $\mathrm{x}$ & $\mathrm{x}$ & & & $\mathrm{x}$ & $\mathrm{x}$ & & \\
\hline Justicia thunbergioides (Lindau) Leonard & 421 & arb & & & & & $\mathrm{x}$ & $\mathrm{x}$ & $\mathrm{x}$ & & \\
\hline Ruellia cf. brevifolia (Pohl) C.Ezcurra & 3 & arb & & $\mathrm{x}$ & $\mathrm{x}$ & $\mathrm{x}$ & & $\mathrm{x}$ & $\mathrm{x}$ & & \\
\hline Ruellia discifolia Oliver & 858 & arb & & & & & & & & & \\
\hline \multicolumn{12}{|l|}{ ALSTROEMERIACEAE } \\
\hline Alstroemeria sp. 1 & 26 & erva & & & & & & & & & \\
\hline Alstroemeria $\mathrm{sp} .2$ & 1400 & erva & & & $\mathrm{x}$ & & & & & & \\
\hline Bomarea edulis Herb. & 312 & trep & $\mathrm{x}$ & $\mathrm{x}$ & $\mathrm{x}$ & $\mathrm{x}$ & $\mathrm{x}$ & $\mathrm{x}$ & $\mathrm{x}$ & & $\mathrm{x}$ \\
\hline \multicolumn{12}{|l|}{ AMARANTHACEAE } \\
\hline Alternanthera brasiliana (L.) Kuntze & 444 & erva & $\mathrm{x}$ & $\mathrm{x}$ & $\mathrm{x}$ & & & & & & \\
\hline
\end{tabular}


FAMÍLIA/ESPÉCIE

Alternanthera sp.1

Alternanthera sp.2

Chamissoa acuminata Mart.

Chamissoa altissima (Jacq.) Kunth

Pfaffia sp.1

\section{AMARYLLIDACEAE}

Hippeastrum reticulatum Herb.

\section{ANACARDIACEAE}

Astronium fraxinifolium Schott ex Spreng.

Myracrodruon urundeuva Allemão

Tapirira obtusa (Benth.) J.D. Mitch.

ANNONACEAE

Porcelia macrocarpa (Warm.) R.E. Fr.

\section{APOCYNACEAE}

Asclepias curassavica L.

Aspidosperma cylindrocarpon Müll.Arg.

Aspidosperma parvifolium A. DC.

Forsteronia sp.1

Forsteronia sp.2

Forsteronia sp.3

Marsdenia sp.1

Orthosia scoparia (Nutt.) Liede \& Meve

Oxypetalum banksii Schult.

Oxypetalum erianthum Decne.

Prestonia cf. coalita (Vell.) Woodson

Prestonia cf. riedelii (Müll. Arg.) Markgr.

Prestonia sp.

Prestonia speciosa Donn. Sm.

AQUIFOLIACEAE

Ilex sp.

ARACEAE

Anthurium minarum Sakur. \& Mayo

Anthurium pentaphyllum (Aubl.) G. Don

Anthurium solitarium Schott

Asterostigma lombardii E.G.Gonç. P.H.A

Hábito CA1 CE2 MA3 AM4 CA4 CE4 MA4 PP4 PT4

$\begin{array}{lllllllll}645 & \text { erva } & & & & & & & \\ 415 & \text { erva } & & & & & & & \\ 361 & \text { arb } & \text { x } & \text { x } & \text { x } & & \text { x } & & \text { x } \\ 573 & \text { arb } & & \text { x } & \text { x } & \text { x } & \text { x } & \text { x } & \text { x } \\ 96 & \text { erva } & & & & & & & \end{array}$

30 erva

$\mathrm{X}$

$\mathrm{X}$

$\begin{array}{ccccccccc}831 & \text { árv } & & \mathrm{x} & \mathrm{x} & \mathrm{x} & & \mathrm{x} & \mathrm{x} \\ 17 & \text { árv } & \mathrm{x} & \mathrm{x} & \mathrm{x} & & \mathrm{x} & \mathrm{x} & \mathrm{x} \\ 1248 & \text { árv } & \mathrm{x} & \mathrm{x} & \mathrm{x} & \mathrm{x} & & \mathrm{x} & \mathrm{x}\end{array}$

34 árv $\quad \mathrm{x} \quad \mathrm{x} \quad \mathrm{x}$

\begin{tabular}{|c|c|c|c|c|c|c|c|}
\hline 825 arb & $\mathrm{x}$ & $\mathrm{x}$ & $\mathrm{x}$ & $\mathrm{x}$ & $\mathrm{x}$ & & $\mathrm{x}$ \\
\hline árv & & $\mathrm{x}$ & $\mathrm{x}$ & $\mathrm{x}$ & & $\mathrm{x}$ & $\mathrm{x}$ \\
\hline 1345 & $\mathrm{x}$ & $\mathrm{x}$ & $\mathrm{x}$ & $\mathrm{x}$ & $\mathrm{x}$ & $\mathrm{x}$ & $\mathrm{x}$ \\
\hline 1714 trep & & & & & & & \\
\hline trep & & & & & & & \\
\hline trep & & & & & & & \\
\hline trep & & & & & & & \\
\hline erva & & & & & & & $\mathrm{x}$ \\
\hline 1015 & & $\mathrm{x}$ & $\mathrm{x}$ & & & $\mathrm{x}$ & $\mathrm{x}$ \\
\hline 1145 & & $\mathrm{x}$ & $\mathrm{x}$ & & & $\mathrm{x}$ & $\mathrm{x}$ \\
\hline trep & $\mathrm{x}$ & $\mathrm{x}$ & $\mathrm{x}$ & $\mathrm{x}$ & $\mathrm{x}$ & $\mathrm{x}$ & $\mathrm{x}$ \\
\hline trep & & $\mathrm{x}$ & $\mathrm{x}$ & & & $\mathrm{x}$ & $\mathrm{x}$ \\
\hline trep & & & & & & & \\
\hline trep & & & & & & & \\
\hline 1237 árv & & & & & & & \\
\hline erva & & $\mathrm{x}$ & $\mathrm{x}$ & & & $\mathrm{x}$ & $\mathrm{x}$ \\
\hline erva & $\mathrm{x}$ & $\mathrm{x}$ & $\mathrm{x}$ & $\mathrm{x}$ & & & $\mathrm{x}$ \\
\hline erva & & $\mathrm{x}$ & $\mathrm{x}$ & $\mathrm{x}$ & $\mathrm{x}$ & $\mathrm{x}$ & $\mathrm{x}$ \\
\hline erva & & & $\mathrm{x}$ & & & & $\mathrm{x}$ \\
\hline
\end{tabular}


FAMÍLIA/ESPÉCIE

Philodendron bipinnatifidum Schott

Philodendron brasiliense Engl.

ARECACEAE

Syagrus sp.

\section{ARISTOLOCHIACEAE}

Aristolochia cf. papillaris Mast.

ASTERACEAE

Acmella brachyglossa Cass.

Ageratum conyzoides L.

Bidens riparia Kunth

Blainvillea $\mathrm{cf}$. dichotoma (Murray) Stewart

Chaptalia nutans (L.) Polak.

Chromolaena maximilianii (Schrad. ex DC.)

R.M.King \& H.Rob.

Cosmos caudatus Kunth

Dasyphyllum sp.

Delilia biflora (L.) Kuntze

Elephantopus mollis Kunth

Emilia sonchifolia (L.) DC.

Eupatorium sp.

Gardnerina angustata (Gardner) R.M. King \& H. Rob.

Jungia sp.

Lepidaploa helophila (Mart. ex DC.) H. Rob.

Lepidaploa remotiflora (Rich.) H. Rob.

Lepidaploa $\mathrm{sp}$.

Mikania sp.

Pentacalia desiderabilis (Vell) Cuatrec.

Praxelis sp.

Stifftia parviflora (Leandro) D.Don

Trixis sp.

Vernonanthura ferruginea (Less.) H. Rob.

Viguiera $\mathrm{sp}$

Wedelia $\mathrm{sp}$.

BEGONIACEAE
Hábito CA1 CE2 MA3 AM4 CA4 CE4 MA4 PP4 PT4

$\begin{array}{llllllll}384 & \text { erva } & x & x & x & x & x & x \\ 1407 & \text { erva } & & \text { x } & \text { x } & & & \end{array}$

1327 árv

$1190 \quad$ trep $\quad \mathrm{x} \quad \mathrm{x} \quad \mathrm{x} \quad \mathrm{x} \quad \mathrm{x} \quad \mathrm{x}$

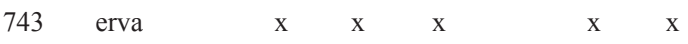

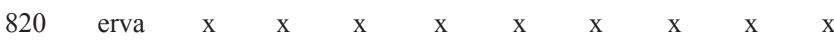

595 erva $\quad \mathrm{x}$

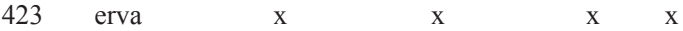

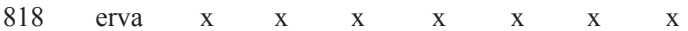

514 erva $\quad \mathrm{x} \quad \mathrm{x} \quad \mathrm{x} \quad \mathrm{x} \quad \mathrm{x}$

570 erva $x$

15 arb

596 erva x

$\begin{array}{lllllllllllll}447 & \text { erva } & \mathrm{x} & \mathrm{x} & \mathrm{x} & \mathrm{x} & \mathrm{x} & \mathrm{x} & \mathrm{x} & \mathrm{x} & \mathrm{x}\end{array}$

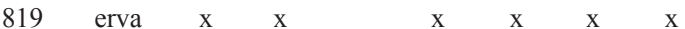

$31 \quad$ arb

1366 erva $\quad \mathrm{x}$

607 erva

737 erva

1133 erva $\mathrm{x}$

435 erva

9 trep

1144 arb

736 erva

829 árv

626 erva

784 arb

539 erva

436 erva

Begonia fischeri Schrank

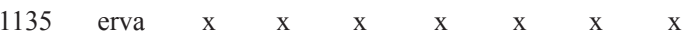


FAMÍLIA/ESPÉCIE

Begonia reniformis Dryand.

Begonia sp.1

Begonia sp.2

\section{BIGNONIACEAE}

Adenocalymma sp.

Bignonia binata Thunb.

Bignonia cf. binata Thunb.

Bignonia sp.

Dolichandra unguis-cati (L.) L.G.Lohmann

Fridericia speciosa Mart.

Handroanthus cf. impetiginosus Mattos

Handroanthus serratifolius (Vahl) S.O.Grose

Pyrostegia venusta Miers

Saritaea triplinervia (Mart. ex DC.) Dugand

Tabebuia sp.

BORAGINACEAE

Cordia tetrandra Aubl.

Cordia trichotoma (Vell.) Arráb. ex Steud.

Heliotropium cf. angiospermum Murray

Heliotropium cf. filiforme Lehm.

Heliotropium indicum $\mathrm{L}$.

Tournefortia paniculata Vent.

Tournefortia sp.1

Tournefortia $\mathrm{sp} .2$

Varronia urticifolia (Cham.) J.S. Mill.

\section{BROMELIACEAE}

Acanthostachys strobilacea (Schult. \& Schult.f.) Klotzsch

Aechmea bromeliaefolia (Rudge) Baker

Aechmea distichantha Lem.

Billbergia amoena Lindl.

Billbergia cf. zebrina (Herb.) Lindl.

Billbergia distachia (Vell.) Mez

Pseudananas sagenarius (Arruda) Camargo

Tillandsia loliacea Mart. ex Schult. \& Schult.f.

Tillandsia pohliana Mez
Hábito CA1 CE2 MA3 AM4 CA4 CE4 MA4 PP4 PT4

$\begin{array}{llllllll}391 & \text { erva } & x & x & x & x & x & x \\ 1125 & \text { erva } & & & & & & \\ 336 & \text { erva }\end{array}$

589 trep

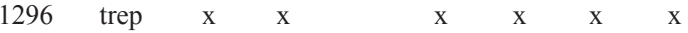

204 trep $\quad$ x $\quad$ x $\quad$ x $\quad$ x $\quad$ x

1018 trep

$\begin{array}{lllllllllllll}99 & \text { trep } & \mathrm{x} & \mathrm{x} & \mathrm{x} & \mathrm{x} & \mathrm{x} & \mathrm{x} & \mathrm{x} & \mathrm{x}\end{array}$

$905 \quad \operatorname{trep} \quad \mathrm{x} \quad \mathrm{x} \quad \mathrm{x} \quad \mathrm{x} \quad \mathrm{x}$

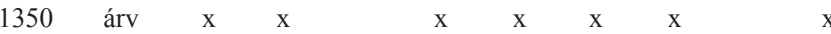

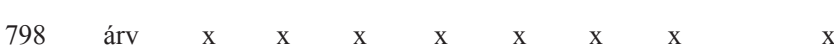

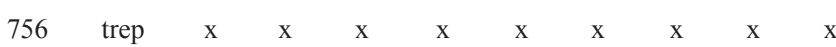

$\begin{array}{llllllllllll}425 & \text { trep } & \mathrm{x} & \mathrm{x} & \mathrm{x} & \mathrm{x} & \mathrm{x} & \mathrm{x} & & & \end{array}$

918 árv

\begin{tabular}{|c|c|c|c|c|c|c|c|c|c|}
\hline 544 árv & & & & $\mathrm{x}$ & & & & & \\
\hline 335 & $\mathrm{x}$ & $\mathrm{x}$ & $\mathrm{x}$ & $\mathrm{x}$ & $\mathrm{x}$ & $\mathrm{x}$ & & & \\
\hline erva & $\mathrm{x}$ & $\mathrm{x}$ & $\mathrm{x}$ & & $\mathrm{x}$ & & $\mathrm{x}$ & & \\
\hline erva & & $\mathrm{x}$ & $\mathrm{x}$ & & & & & & \\
\hline erva & $\mathrm{x}$ & $x$ & $\mathrm{X}$ & $\mathrm{x}$ & $\mathrm{X}$ & $\mathrm{x}$ & $\mathrm{x}$ & $\mathrm{x}$ & $\mathrm{x}$ \\
\hline arb & $\mathrm{x}$ & & $\mathrm{x}$ & $\mathrm{x}$ & $\mathrm{x}$ & & $\mathrm{x}$ & & \\
\hline arb & & & & & & & & & \\
\hline 1050 arb & & & & & & & & & \\
\hline 1078 & & $\mathrm{x}$ & & & & $\mathrm{x}$ & $\mathrm{x}$ & & \\
\hline erva & & $\mathrm{x}$ & $\mathrm{x}$ & & & $\mathrm{x}$ & $\mathrm{x}$ & & \\
\hline erva & $\mathrm{x}$ & $\mathrm{x}$ & $\mathrm{X}$ & $\mathrm{x}$ & $\mathrm{x}$ & $\mathrm{x}$ & $\mathrm{x}$ & & \\
\hline erva & & $\mathrm{x}$ & $\mathrm{x}$ & & & $\mathrm{x}$ & $\mathrm{x}$ & & \\
\hline erva & $\mathrm{x}$ & $\mathrm{x}$ & $\mathrm{x}$ & & $\mathrm{x}$ & $\mathrm{x}$ & $\mathrm{x}$ & & \\
\hline erva & $\mathrm{x}$ & & $\mathrm{X}$ & & & & $\mathrm{x}$ & & \\
\hline erva & & $\mathrm{x}$ & $\mathrm{X}$ & & & $\mathrm{x}$ & $\mathrm{x}$ & & \\
\hline erva & $\mathrm{x}$ & $\mathrm{x}$ & $\mathrm{x}$ & & & $\mathrm{x}$ & $\mathrm{x}$ & & \\
\hline erva & $\mathrm{x}$ & $\mathrm{x}$ & $\mathrm{X}$ & & $\mathrm{x}$ & $\mathrm{x}$ & $\mathrm{x}$ & & \\
\hline erva & $\mathrm{x}$ & $\mathrm{x}$ & $\mathrm{X}$ & & $\mathrm{x}$ & $\mathrm{x}$ & $\mathrm{x}$ & & \\
\hline
\end{tabular}




\section{FAMÍLIA/ESPÉCIE}

Tillandsia polystachia (L.) L.

Tillandsia streptocarpa Baker

Tillandsia tenuifolia var. vaginata (Wawra)L.B.Sm.

Vriesa $\mathrm{sp}$.

\section{CACTACEAE}

Brasiliopuntia brasiliensis (Wilk.)A. Berger

Cereus jamacaru ssp. calcirupicula (F. Ritter) N.P. Taylor \& Zappi

Epiphyllum phyllanthus (L.) Haw.

Hylocereus setaceus (Salm-Dyck) Ralf Bauer

Pereskia aculeata Milbr.

\section{CANNABACEAE}

Celtis iguanaea (Jacq.) Sarg.

Celtis pubescens (Kunth) Spreng.

Trema micrantha (L.) Blume

\section{CANNACEAE}

Canna indica $\mathrm{L}$.

\section{CARICACEAE}

Vasconcellea quercifolia A.St.-Hil.

\section{CELASTRACEAE}

Hippocratea volubilis L.

Maytenus robusta Reissek

CLEOMACEAE

Cleome hassleriana Chodat

Cleome viridiflora Schreb.

\section{COMBRETACEAE}

Combretum laxum Jacq.

Terminalia glabrescens Mart.

COMMELINACEAE

Commelina diffusa Burm.F.

Dichorisandra hexandra Standl.

Gibasis geniculata (Jacq.) Rohweder

Tradescantia zanonia (L.) Sw.

Tripogandra elata D.R.Hunt

CONNARACEAE
Melo, P.H.A

Hábito CA1 CE2 MA3 AM4 CA4 CE4 MA4 PP4 PT4

$\begin{array}{ccccccccc}412 & \text { erva } & \text { x } & \text { x } & \text { x } & & \text { x } & \text { x } & \text { x } \\ 94 & \text { erva } & \text { x } & \text { x } & \text { x } & & \text { x } & \text { x } & \text { x } \\ 7 & \text { erva } & \text { x } & \text { x } & \text { x } & \text { x } & \text { x } & \text { x } & \text { x } \\ 2419 & \text { erva } & & & & & & & \end{array}$

\author{
67
}

933 arb $\quad \mathrm{x} \quad \mathrm{x} \quad \mathrm{x} \quad \mathrm{x}$

$\begin{array}{lllllllll}372 & \text { erva } & \mathrm{x} & \mathrm{x} & \mathrm{x} & \mathrm{x} & \mathrm{x} & \mathrm{x}\end{array}$

$\begin{array}{llllllllllll}78 & \text { erva } & \mathrm{x} & \mathrm{x} & \mathrm{x} & \mathrm{x} & \mathrm{x} & \mathrm{x} & \mathrm{x}\end{array}$

$\begin{array}{lllllllll}485 & \text { trep } & \mathrm{x} & \mathrm{x} & \mathrm{x} & \mathrm{x} & \mathrm{x}\end{array}$

$\begin{array}{cccccccccc}55 & \text { árv } & \mathrm{x} & \mathrm{x} & \mathrm{x} & \mathrm{x} & \mathrm{x} & \mathrm{x} & \mathrm{x} & \mathrm{x} \\ 1737 & \text { árv } & \mathrm{x} & & & \mathrm{x} & \mathrm{x} & \mathrm{x} & & \mathrm{x} \\ 285 & \text { árv } & \mathrm{x} & \mathrm{x} & \mathrm{x} & \mathrm{x} & \mathrm{x} & \mathrm{x} & \mathrm{x} & \mathrm{x}\end{array}$

$\begin{array}{lllllllll}1534 & \operatorname{arb} & \mathrm{x} & \mathrm{x} & \mathrm{x} & & \mathrm{x} & \mathrm{x}\end{array}$

$\begin{array}{lllllllll}382 & \text { trep } & \mathrm{x} & \mathrm{x} & \mathrm{x} & \mathrm{x} & \mathrm{x} & \mathrm{x} & \end{array}$

$\begin{array}{lllllllll}56 & \operatorname{arb} & \mathrm{x} & \mathrm{x} & \mathrm{x} & \mathrm{x} & \mathrm{x} & \mathrm{x} & \mathrm{x}\end{array}$

879 arb $\quad \mathrm{x} \quad \mathrm{x}$

$\begin{array}{llllll}533 & \operatorname{arb} & \mathrm{x} & \mathrm{x} & \mathrm{x} & \mathrm{x}\end{array}$

$\begin{array}{llllllllll}669 & \operatorname{arb} & \mathrm{x} & \mathrm{x} & \mathrm{x} & \mathrm{x} & \mathrm{x} & \mathrm{x} & \mathrm{x}\end{array}$

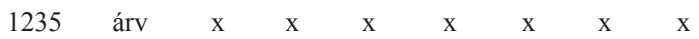

$\begin{array}{llllllll}583 & \text { erva } & \text { x } & \text { x } & \text { x } & \text { x } & \text { x } & \text { x } \\ 253 & \text { erva } & \text { x } & \text { x } & \text { x } & \text { x } & \text { x } & \text { x } \\ 546 & \text { erva } & \text { x } & & & \text { x } & & \text { x } \\ 266 & \text { erva } & & & \text { x } & & & \text { x } \\ 481 & \text { erva } & & & & & \end{array}$

Rourea sp.

909 árv

Rodriguésia 64(1): A1-A16. 2013 
FAMÍLIA/ESPÉCIE

Melo, P.H.A

CONVOLVULACEAE

Dichondra sp.

Ipomoea sp.

Ipomoea tubata Nees

Jacquemontia sp.

Merremia sp.

CUCURBITACEAE

Sicyos warmingii Cogn.

Wilbrandia hibiscoides Silva Manso

CYPERACEAE

Cyperus sp.

Eleocharis sp.

Rhynchopera sp.

\section{DIOSCOREACEAE}

Dioscorea cf. asperula Pedralli

Dioscorea cf. piperifolia Humb. \& Bonpl. ex Willd.

Dioscorea $\mathrm{sp} .1$

Dioscorea $\mathrm{sp.2}$

Dioscorea sp.3

\section{ERYTHROXYLACEAE}

Erythroxylum strobilaceum Peyr.

EUPHORBIACEAE

Acalypha cf. diversifolia Jacq.

Acalypha cf. gracilis Spreng.

Acalypha communis Müll.Arg.

Actinostemon klotzschii (Didr.) Pax

Cnidoscolus cf. urens (L.) Arthur

Cnidoscolus lombardii Fern.Casas

Croton celtidifolius Baill.

Croton macrobotrys Baill.

Croton rottlerifolius Baill.

Croton triqueter Lam.

Croton urucurana Baill.

Dalechampia stipulacea Müll.Arg.

Euphorbia comosa Vell.

22

339
Hábito CA1 CE2 MA3 AM4 CA4 CE4 MA4 PP4 PT4

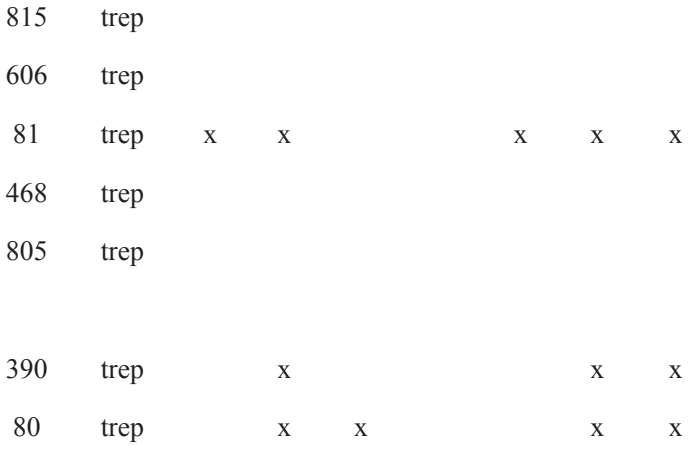

998 erva

1808 trep

398 trep

1086 trep

59 arb $\quad x \quad x$

$\begin{array}{lllllllll}1136 & \text { arb } & & \text { x } & & \text { x } & & \text { x } & \\ 431 & \text { arb } & & \text { x } & \text { x } & \text { x } & \text { x } & \text { x } & \text { x } \\ 289 & \text { arb } & \text { x } & \text { x } & \text { x } & & \text { x } & \text { x } & \text { x } \\ 69 & \text { arb } & \text { x } & \text { x } & \text { x } & & & & \text { x } \\ 12 & \text { arb } & \text { x } & \text { x } & \text { x } & & & \\ 249 & \text { arb } & & & & & & & \end{array}$

953 árv $\mathrm{x}$ x

29 árv $\quad x$

261 arb $\quad x \quad x \quad$ x $\quad x$

$298 \quad \operatorname{arb} \quad \mathrm{x} \quad \mathrm{x} \quad \mathrm{x}$

207 árv x x

65 trep $\quad x \quad$ x

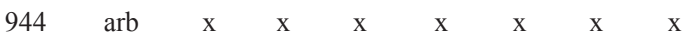


FAMÍLIA/ESPÉCIE

Euphorbia heterophylla L.

Euphorbia hirta L.

Euphorbia sciadophila Boiss.

Jatropha sp.

Manihot cf. grahamii Hook.

Pachystroma longifolium (Nees)

Romanoa tamnoides (A.Juss.) Radcl.-Sm.

Sebastiania brasiliensis Spreng.

Tragia sp.

Tragia volubilis L.

FABACEAE

Acacia polyphylla DC.

Acacia sp.

Albizia niopoides (Spruce ex Benth.) Burkart

Anadenanthera colubrina (Vell.) Brenan

Andira vermifuga (Mart.) Benth.

Bauhinia longifolia (Bong.) D.Dietr.

Bauhinia sp.

Bionia bella Mart. ex Benth.

Calliandra foliolosa Benth.

Centrolobium microchaete (Mart. ex Benth.)

H.C.Lima

Centrolobium tomentosum Guillem. ex Benth.

Centrosema sagittatum (Humb. \& Bonpl. ex Willd.)

Brandegee

Chamaecrista sp.

Chloroleucon tortum (Mart.) Pittier

Crotalaria cf. incana L.

Dialium sp.

Dioclea sp.

Enterolobium contortisiliquum (Vell.) Morong

Erythrina crista-galli L.

Erythrina verna Vell.

Holocalyx balansae Micheli

Hymenaea courbaril L.

Inga marginata Willd. P.H.A

Hábito CA1 CE2 MA3 AM4 CA4 CE4 MA4 PP4 PT4

\begin{tabular}{|c|c|c|c|c|c|c|c|}
\hline 417 & arb & $\mathrm{x}$ & & $\mathrm{x}$ & $\mathrm{x}$ & $\mathrm{x}$ & \\
\hline 823 & erva & & $\mathrm{x}$ & & $\mathrm{X}$ & $\mathrm{x}$ & $\mathrm{X}$ \\
\hline 432 & erva & $\mathrm{x}$ & & $\mathrm{x}$ & $\mathrm{x}$ & $\mathrm{X}$ & $\mathrm{x}$ \\
\hline 2459 & árv & & & & & & \\
\hline 54 & arb & & & $\mathrm{x}$ & & & \\
\hline 750 & árv & & & $\mathrm{x}$ & & $\mathrm{X}$ & $\mathrm{X}$ \\
\hline 265 & trep & $\mathrm{X}$ & $\mathrm{X}$ & $\mathrm{X}$ & $\mathrm{X}$ & $\mathrm{X}$ & $\mathrm{X}$ \\
\hline 68 & árv & $\mathrm{x}$ & $\mathrm{X}$ & $\mathrm{X}$ & & & \\
\hline 470 & trep & & & & & & \\
\hline 555 & trep & $\mathrm{x}$ & & $\mathrm{x}$ & $\mathrm{x}$ & $\mathrm{x}$ & \\
\hline
\end{tabular}$$
23
$$

576

1062 árv

229 árv

1520 árv

48 ár

630 trep

1192 trep

635 arb

1356 árv

210 árv

1049 trep

429

erva

900 árv

734

368

503

467

813 árv

2447 árv

215 árv

251 árv

1233 árv $\operatorname{arb} \quad \mathrm{x}$

rv

ran

$\mathrm{X} \quad \mathrm{X}$

r

$\mathrm{X}$

$\mathrm{p}$

ep

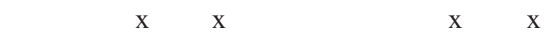

ep

X $\mathrm{X}$ X

rv $\quad x \quad x \quad x$ $\begin{array}{llllllllllll}\text { arb } & \mathrm{x} & \mathrm{x} & \mathrm{x} & \mathrm{X} & \mathrm{x} & \mathrm{x} & \mathrm{x} & \mathrm{x} & \mathrm{x}\end{array}$ árv rep

$\begin{array}{lllllll}x & x & x & x & x\end{array}$

$\begin{array}{llll}x & x & x & x\end{array}$

$\begin{array}{llllll} & x & x & x & X & X\end{array}$

$\begin{array}{llllll} & x & x & x & x & x\end{array}$

$\begin{array}{llllllll} & \mathrm{x} & \mathrm{x} & \mathrm{x} & \mathrm{x} & \mathrm{x} & \mathrm{x} & \mathrm{x}\end{array}$ $\mathrm{x} \quad \mathrm{x}-\mathrm{x}$ 
FAMÍLIA/ESPÉCIE

Inga vera Willd.

Lonchocarpus cf. muehlbergianus Hassl.

Lonchocarpus cultratus (Vell.) Az.-Tozzi \& H.C.Lima

Lonchocarpus sericeus (Poir.) DC.

Machaerium hirtum (Vell.) Stellfeld

Machaerium villosum Vogel

Mimosa sp.1

Mimosa sp.2

Myroxylon peruiferum L.f.

Platypodium elegans Vogel

Senegalia tenuifolia (L.) Britton \& Rose

Senna multijuga (L.C.Rich.) H.S.Irwin \& Barneby

Vachellia farnesiana (L.) Wight \& Arn.

Zollernia glabra (Spreng.) Yakovlev

GESNERIACEAE

Chautemsia calcicola A.O.Araujo \& V.C.Souza

Sinningia aggregata (Ker Gawl.) Wiehler

Sinningia conspicua (Seem.) G. Nicholson

Sinningia warmingii (Hiern) Chautems

IRIDACEAE

Neomarica cf. glauca (Seub. ex Klatt) Sprague

LAMIACEAE

Aegiphila cf. vitelliniflora Walp.

Hyptis mutabilis (Rich.) Briq.

Ocimum cf. carnosum (Spreng.) Link \& Otto ex Benth.

\section{LAURACEAE}

Aniba intermedia (Meisn.) Mez

Nectandra cf. membranacea (Sw.) Griseb.

Nectandra cf. oppositifolia Nees

Nectandra megapotamica (Spreng.) Mez

Nectandra nitidula Nees

Ocotea corymbosa (Meisn.) Mez

\section{LOASACEAE}

Hábito CA1 CE2 MA3 AM4 CA4 CE4 MA4 PP4 PT4 P.H.A

\begin{tabular}{|c|c|c|c|c|c|c|c|c|}
\hline 49 & árv & & $\mathrm{x}$ & $\mathrm{x}$ & $\mathrm{x}$ & & $\mathrm{x}$ & $\mathrm{x}$ \\
\hline 1782 & árv & & $\mathrm{x}$ & $\mathrm{x}$ & & & & $\mathrm{x}$ \\
\hline 516 & árv & $\mathrm{x}$ & $\mathrm{x}$ & $\mathrm{x}$ & $\mathrm{x}$ & $\mathrm{x}$ & $\mathrm{x}$ & $\mathrm{x}$ \\
\hline 979 & árv & $\mathrm{x}$ & $\mathrm{x}$ & $\mathrm{x}$ & $\mathrm{x}$ & $\mathrm{x}$ & $\mathrm{x}$ & $\mathrm{x}$ \\
\hline 1088 & árv & $\mathrm{x}$ & & $\mathrm{x}$ & $\mathrm{x}$ & $\mathrm{x}$ & $\mathrm{x}$ & $\mathrm{x}$ \\
\hline 1091 & árv & & $\mathrm{x}$ & $\mathrm{x}$ & & $\mathrm{x}$ & $\mathrm{x}$ & $\mathrm{x}$ \\
\hline 471 & trep & & & & & & & \\
\hline 1073 & trep & & & & & & & \\
\hline 772 & árv & & $\mathrm{x}$ & $\mathrm{x}$ & & & $\mathrm{x}$ & $\mathrm{x}$ \\
\hline 912 & árv & $\mathrm{x}$ & $\mathrm{x}$ & $x$ & $\mathrm{x}$ & $\mathrm{x}$ & $\mathrm{x}$ & $\mathrm{x}$ \\
\hline 949 & árv & $\mathrm{x}$ & & & $\mathrm{x}$ & $\mathrm{x}$ & $\mathrm{x}$ & $\mathrm{x}$ \\
\hline 814 & árv & $\mathrm{x}$ & $\mathrm{x}$ & $\mathrm{x}$ & $\mathrm{x}$ & $\mathrm{x}$ & $\mathrm{x}$ & $x$ \\
\hline 1038 & arb & $\mathrm{x}$ & $\mathrm{x}$ & & $\mathrm{x}$ & $\mathrm{x}$ & & $\mathrm{x}$ \\
\hline
\end{tabular}

\begin{tabular}{|c|c|c|c|c|c|c|c|c|c|}
\hline 408 & erva & & & & & & $\mathrm{x}$ & & \\
\hline 1421 & erva & $\mathrm{x}$ & $\mathrm{x}$ & $\mathrm{x}$ & & & $\mathrm{x}$ & $\mathrm{x}$ & \\
\hline 1815 & erva & & & $\mathrm{x}$ & & & & $\mathrm{x}$ & \\
\hline 344 & erva & & $\mathrm{x}$ & $\mathrm{x}$ & & & $\mathrm{x}$ & $\mathrm{x}$ & \\
\hline 1178 & erva & & & $\mathrm{x}$ & & & $\mathrm{X}$ & $\mathrm{x}$ & \\
\hline 307 & árv & $\mathrm{x}$ & $\mathrm{x}$ & $\mathrm{x}$ & $\mathrm{x}$ & $\mathrm{x}$ & x & $\mathrm{X}$ & \\
\hline 584 & arb & $\mathrm{x}$ & $\mathrm{x}$ & $\mathrm{x}$ & $\mathrm{x}$ & $\mathrm{x}$ & $\mathrm{x}$ & $\mathrm{x}$ & $\mathrm{X}$ \\
\hline 739 & erva & $\mathrm{x}$ & & $\mathrm{x}$ & & & & $\mathrm{x}$ & \\
\hline
\end{tabular}

$\begin{array}{lllllllll}1202 & \text { árv } & & & & & & & \mathrm{x} \\ 2442 & \text { árv } & \mathrm{x} & \mathrm{x} & \mathrm{x} & \mathrm{x} & \mathrm{x} & \mathrm{x} & \mathrm{x} \\ 624 & \text { árv } & \mathrm{x} & \mathrm{x} & \mathrm{x} & & \mathrm{x} & \mathrm{x} & \mathrm{x} \\ 520 & \text { árv } & & \mathrm{x} & \mathrm{x} & & & \mathrm{x} & \mathrm{x} \\ 1351 & \text { árv } & \mathrm{x} & \mathrm{x} & \mathrm{x} & \mathrm{x} & \mathrm{x} & \mathrm{x} \\ 2416 & \text { árv } & \mathrm{x} & \mathrm{x} & \mathrm{x} & & \mathrm{x} & \mathrm{x} \\ & & & & & & & \\ 2462 & \text { arb } & & & & & & & \end{array}$


FAMÍLIA/ESPÉCIE

Mentzelia sp.

LOGANIACEAE

Strychnos cf. gardneri A. DC.

\section{MALPIGHIACEAE}

Banisteriopsis sp.

Dicella bracteosa (A. Juss.) Griseb.

Heteropterys sp.

Tetrapterys chamaecerasifolia A Juss.

Tetrapterys sp.

MALVACEAE

Abutilon fluviatile (Vell.)K.Schum.

Bastardia bivalvis (Cav.) H.B.K

Bastardiopsis densiflora (Hook. \& Arn.) Hassl.

Byttneria catalpifolia subsp. sidaefolia (J.St.-Hil.)

Cristóbal

Byttneria sp.

Ceiba pubiflora (A. St.-Hil.) K. Schum.

Corchorus hirtus L.

Gaya gracilipes K. Schum.

Guazuma ulmifolia Lam.

Helicteres ovata Lam.

Luehea divaricata Mart.

Luehea paniculata Mart. \& Zucc.

Malvastrum americanum (L.) Torrey

Melochia argentina R.E.Fries

Pseudabutilon aristulosum (K.Schum.) Krapov.

Pseudobombax sp.

Quararibea floribunda K.Schum.

Sida sp.

Sida urens

Triumfetta cf. abutiloides A.St.Hil.

Triumfetta cf. semitriloba Jacq.

Triumfetta sp.

Wissadula amplissima (L.) R.E. Fr.

Wissadula cf. macrantha R.E.Fries
Hábito CA1 CE2 MA3 AM4 CA4 CE4 MA4 PP4 PT4 erva

rva

$459 \quad$ trep

$\begin{array}{llllllll}406 & \text { trep } & \mathrm{x} & \mathrm{x} & \mathrm{x} & \mathrm{x} & \mathrm{x} & \mathrm{x}\end{array}$

1700 trep

256 tre

$588 \quad$ trep

$888 \quad$ arb

613 arb

774 árv

852 trep

19 arb

542 árv

999 arb

313

82

884

374

2448

620

303

732

$\begin{array}{llllllll} & \mathrm{x} & \mathrm{x} & \mathrm{x} & \mathrm{x} & \mathrm{x} & \mathrm{x} & \mathrm{x}\end{array}$


FAMÍLIA/ESPÉCIE

Melo, P.H.A

MARANTACEAE

Calathea longifolia Klotzsch

Calathea sp.1

Calathea sp.2

Maranta sp.1

Maranta sp.2

MELASTOMATACEAE

Clidemia hirta D.Don

MELIACEAE

Cedrela fissilis Vell.

Cedrela odorata $\mathrm{L}$.

Guarea guidonia (L.) Sleumer

Guarea kunthiana A.Juss.

Guarea macrophylla Vahl

Trichilia catigua A.Juss.

Trichilia cf. casaretti C.DC.

Trichilia clausseni C.DC.

Trichilia elegans A.Juss

Trichilia hirta $\mathrm{L}$.

MENISPERMACEAE

Cissampelos sp.

1299 trep

MORACEAE

Dorstenia caatingae R.M.Castro

Dorstenia cayapia Vell.

Dorstenia vitifolia Gardner

Ficus cf. obtusifolia (Miq.) Miq.

Ficus citrifolia Mill.

Ficus sp.

Maclura tinctoria (L.) D.Don ex Steud.

MYRSINACEAE

Ardisia guianensis (Aubl.) Mez

Myrsine umbellata Mart.

MYRTACEAE

Campomanesia cf. guaviroba (DC.) Kiaersk.

Eugenia acutata Miq.

872

$\operatorname{arb}$

371 árv
Hábito CA1 CE2 MA3 AM4 CA4 CE4 MA4 PP4 PT4

$\begin{array}{ll}269 & \text { erva } \\ 228 & \text { erva } \\ 530 & \text { erva } \\ 1802 & \text { erva } \\ 314 & \text { erva }\end{array}$

$\begin{array}{lllllllll}50 & \text { árv } & \mathrm{x} & \mathrm{x} & \mathrm{x} & \mathrm{x} & \mathrm{x} & \mathrm{x} & \mathrm{x}\end{array}$

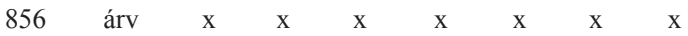

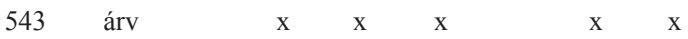

$\begin{array}{lllllllll}202 & \text { árv } & \mathrm{x} & \mathrm{x} & \mathrm{x} & \mathrm{x} & \mathrm{x} & \mathrm{x} & \mathrm{x}\end{array}$

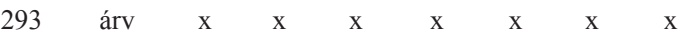

286 árv $\mathrm{x} \quad \mathrm{x} \quad \mathrm{x}$

20 árv $\quad \mathrm{x} \quad \mathrm{x} \quad \mathrm{x} \quad \mathrm{x}$

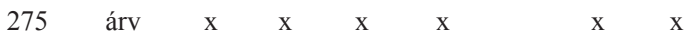

$\begin{array}{lllllllll}1072 & \text { árv } & \mathrm{x} & \mathrm{x} & \mathrm{x} & \mathrm{x} & \mathrm{x} & \mathrm{x} & \mathrm{x}\end{array}$

$\begin{array}{lllllllllll}1288 & \text { erva } & \text { x } & & \text { x } & & \text { x } & & \text { x } & \\ 370 & \text { erva } & \text { x } & & \text { x } & & \text { x } & \text { x } & \text { x } & \\ 1867 & \text { erva } & & \text { x } & & & & & & \\ 30 & \text { árv } & \text { x } & \text { x } & \text { x } & & & \text { x } & \text { x } & \text { x } \\ 247 & \text { árv } & \text { x } & & \text { x } & \text { x } & \text { x } & \text { x } & \text { x } & \\ 1834 & \text { árv } & & & & & & & & & \\ 453 & \text { árv } & \text { x } & & \text { x } & \text { x } & \text { x } & \text { x } & \text { x } & \text { x } & \text { x }\end{array}$

$\begin{array}{ccccccccc}666 & \text { árv } & & & & \text { x } & & & \text { x } \\ 1254 & \text { árv } & \text { x } & \text { x } & \text { x } & \text { x } & \text { x } & \text { x } & \text { x }\end{array}$

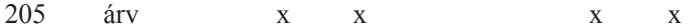

920 arb $x \quad x \quad x$ 
FAMÍLIA/ESPÉCIE

Eugenia cf. ligustrina (Sw.) Willd.

Eugenia florida DC.

Eugenia lagoensis Kiaersk.

Eugenia repanda $\mathrm{O}$.Berg

Eugenia sp.

Siphoneugena sp.

NYCTAGINACEAE

Bougainvillea spectabilis Willd.

Guapira opposita (Vell.) Reitz

Mirabilis jalapa $\mathrm{L}$.

Pisonia aculeata $\mathrm{L}$.

Acianthera aphthosa (Lindl.) Pridgeon \& M.W.Chase

Catasetum fimbriatum Rchb.f.

Cattleya bicolor Lindl.

Cattleya walkeriana Gardner

Cohniella cebolleta (Jacq.) Christenson

Cranichis candida (Barb.Rodr.) Cogn.

Cyclopogon longibracteatus (Barb. Rodr.) Schltr.

Cyrtopodium glutiniferum Raddi

Epidendrum anceps Sw.

Epidendrum paniculatum Sessé \& Moc.

Galeandra hysterantha Barb. Rodr.

Ionopsis utricularioides (Sw.) Lindl.

Isochilus linearis (Ruiz \& Pav.) R.Br. in W.T.Aiton

Leptotes bicolor Lindl.

Lophiaris pumila (Lindl.) Braem

Mesadenella cuspidata (Lindl.) Garay

Miltonia flavescens Lindl.

Myoxanthus lonchophyllus (Barb. Rodr.) Luer

Notylia cf. hemitricha Barb. Rodr.

Octomeria sp.

Oeceoclades maculata (Lindl.) Lindl.

Pleurothallis sp.1

Pleurothallis sp.2
ORCHIDACEAE

Hábito CA1 CE2 MA3 AM4 CA4 CE4 MA4 PP4 PT4 P.H.A

$\begin{array}{ccccccccc}25 & \text { árv } & \mathrm{x} & \mathrm{x} & \mathrm{x} & \mathrm{x} & \mathrm{x} & \mathrm{x} & \mathrm{x} \\ 1735 & \text { árv } & \mathrm{x} & \mathrm{x} & \mathrm{x} & \mathrm{x} & \mathrm{x} & \mathrm{x} & \mathrm{x} \\ 2488 & \text { arb } & & \mathrm{x} & \mathrm{x} & & & \mathrm{x} & \mathrm{x} \\ 561 & \text { árv } & \text { x } & & \mathrm{x} & & & & \mathrm{x} \\ 213 & \text { arb } & & & & & & & \\ 1820 & \text { arb } & & & & & & & \end{array}$

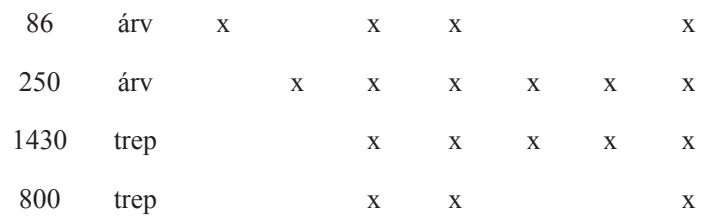

\begin{tabular}{|c|c|c|c|c|c|c|c|c|}
\hline 762 & erva & & & & & & & $\mathrm{x}$ \\
\hline 350 & erva & & $\mathrm{x}$ & $x$ & & & $\mathrm{x}$ & $\mathrm{x}$ \\
\hline 345 & erva & & $\mathrm{x}$ & $\mathrm{x}$ & & & $\mathrm{x}$ & $x$ \\
\hline 1352 & erva & & $\mathrm{x}$ & & $\mathrm{x}$ & & $\mathrm{x}$ & \\
\hline 648 & erva & & $\mathrm{x}$ & & $\mathrm{x}$ & & $\mathrm{x}$ & $x$ \\
\hline 1148 & erva & & $\mathrm{x}$ & $\mathrm{x}$ & & & $\mathrm{x}$ & $\mathrm{x}$ \\
\hline 13 & erva & & & $\mathrm{x}$ & & & & $\mathrm{x}$ \\
\hline 72 & erva & & & $x$ & $\mathrm{x}$ & & & $\mathrm{x}$ \\
\hline 1142 & erva & & $\mathrm{x}$ & & $\mathrm{x}$ & & $\mathrm{x}$ & \\
\hline 58 & erva & & & & & & & \\
\hline 1253 & erva & & & & & & $\mathrm{x}$ & \\
\hline 27 & erva & & $\mathrm{x}$ & $\mathrm{X}$ & $\mathrm{x}$ & & $\mathrm{x}$ & $x$ \\
\hline 1198 & erva & & $\mathrm{x}$ & $\mathrm{X}$ & & & $\mathrm{x}$ & $\mathrm{x}$ \\
\hline 917 & erva & & $\mathrm{x}$ & $\mathrm{x}$ & & & $\mathrm{x}$ & $\mathrm{x}$ \\
\hline 62 & erva & & $\mathrm{x}$ & & & & & $\mathrm{x}$ \\
\hline 1147 & erva & & $\mathrm{x}$ & $\mathrm{X}$ & & & $\mathrm{x}$ & $\mathrm{x}$ \\
\hline 915 & erva & $\mathrm{x}$ & & $\mathrm{x}$ & & $\mathrm{x}$ & & X \\
\hline 347 & erva & & & & & & $\mathrm{x}$ & $\mathrm{x}$ \\
\hline 411 & erva & & $\mathrm{x}$ & $\mathrm{x}$ & & & $\mathrm{x}$ & $\mathrm{x}$ \\
\hline 1341 & erva & & & & & & & \\
\hline 1100 & erva & $\mathrm{x}$ & $\mathrm{x}$ & $\mathrm{x}$ & $\mathrm{x}$ & $\mathrm{x}$ & $\mathrm{x}$ & $\mathrm{x}$ \\
\hline 1684 & erva & & & & & & & \\
\hline 84 & erva & & & & & & & \\
\hline
\end{tabular}

Rodriguésia 64(1): A1-A16. 2013 
FAMÍLIA/ESPÉCIE

P.H.A

Pleurothallis sp. 3

Polystachya concreta (Jacq.) Garay \& H.R. Sweet

Pteroglossa roseoalba (Rchb. f.) Salazar \& M.W. Chase

Sarcoglottis fasciculata (Vell.) Schltr.

Sophronitis cernua Lindl.

OXALIDACEAE

Oxalis cf. rhombeo-ovata A.St.-Hil.

Oxalis sp.

PAPAVERACEAE

Argemone mexicana L.

PASSIFLORACEAE

Passiflora cf. galbana Mast.

Passiflora foetida $\mathrm{L}$.

Passiflora suberosa $\mathrm{L}$.

PHYLLANTHACEAE

Phyllanthus acuminatus Vahl

Phyllanthus sp.

\section{PHYTOLACCACEAE}

Petiveria alliacea $\mathrm{L}$.

Phytolacca dioica $\mathrm{L}$.

Phytolacca thyrsiflora Fenzl ex J.A. Schmidt

PICRAMNIACEAE

Picramnia ramiflora Planch.

\section{PIPERACEAE}

Peperomia alata Ruiz \& Pav.

Peperomia arifolia Miq

Peperomia blanda H.B. \& K.

Peperomia campinasana C.DC.

Peperomia circinnata Link

Peperomia gardneriana Miq.

Peperomia rotundifolia (L.) Kunth

Peperomia rubricaulis A.Dietr.

Peperomia sp.1

Peperomia sp.2

Peperomia sp.3
Hábito CA1 CE2 MA3 AM4 CA4 CE4 MA4 PP4 PT4

1353 erva

\begin{tabular}{|c|c|c|c|c|c|c|c|}
\hline 6 & erva & $\mathrm{x}$ & & $\mathrm{x}$ & $\mathrm{x}$ & $\mathrm{x}$ & $\mathrm{x}$ \\
\hline 728 & erva & & & $\mathrm{x}$ & & & \\
\hline 73 & erva & $\mathrm{x}$ & $\mathrm{x}$ & $\mathrm{x}$ & & $\mathrm{x}$ & $\mathrm{X}$ \\
\hline 26 & erva & & $\mathrm{x}$ & $\mathrm{x}$ & & & $\mathrm{X}$ \\
\hline
\end{tabular}

66 arb $\quad x \quad$ x $\quad x \quad x$

248 erva

$880 \quad$ arb

\begin{tabular}{|c|c|c|c|c|c|c|c|c|}
\hline trep & $\mathrm{X}$ & $\mathrm{X}$ & $X$ & & $\mathrm{X}$ & $\mathrm{X}$ & $\mathrm{X}$ & \\
\hline trep & $\mathrm{X}$ & $\mathrm{X}$ & $X$ & $\mathrm{x}$ & $\mathrm{x}$ & $\mathrm{X}$ & $\mathrm{X}$ & $\mathrm{X}$ \\
\hline trep & $\mathrm{X}$ & $\mathrm{X}$ & $\mathrm{X}$ & & & $\mathrm{X}$ & $\mathrm{X}$ & \\
\hline
\end{tabular}

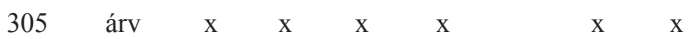

398 erva

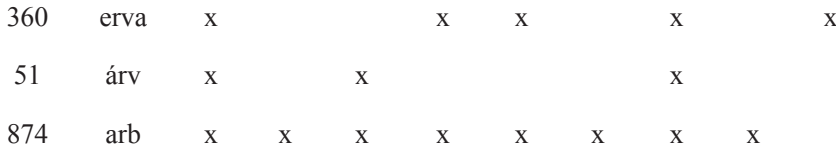

1005 árv $\quad \mathrm{x} \quad \mathrm{x} \quad \mathrm{x}$

$\begin{array}{lllllllll}1833 & \text { erva } & \mathrm{x} & \mathrm{x} & \mathrm{x} & \mathrm{x} & \mathrm{x} & \mathrm{x} & \mathrm{x} \\ 1832 & \text { erva } & & \mathrm{x} & \mathrm{x} & \mathrm{x} & \mathrm{x} & \mathrm{x} \\ 264 & \text { erva } & \mathrm{x} & \mathrm{x} & \mathrm{x} & \mathrm{x} & \mathrm{x} & \mathrm{x} \\ 319 & \text { erva } & \mathrm{x} & \mathrm{x} & \mathrm{x} & & \mathrm{x} & \mathrm{x} \\ 218 & \text { erva } & \mathrm{x} & \mathrm{x} & \mathrm{x} & \mathrm{x} & \mathrm{x} & \mathrm{x} \\ 452 & \text { erva } & & \mathrm{x} & \mathrm{x} & & & \\ 646 & \text { erva } & \mathrm{x} & & \mathrm{x} & \mathrm{x} & & \\ 75 & \text { erva } & \mathrm{x} & & \mathrm{x} & & \mathrm{x} \\ 1189 & \text { erva } & & & & & & \\ 1321 & \text { erva } & & & & & & \\ 671 & \text { erva } & & & & & \end{array}$


FAMÍLIA/ESPÉCIE

Peperomia sp.4

Peperomia trineura Miq.

Piper amalago L.

Piper cf. claussenianum (Miq.) C.DC.

Piper cf. ovatum Vahl

Piper sp.1

Piper sp.2

Piper umbellatum L.

\section{PLANTAGINACEAE}

Stemodia verticillata (Mill.) Hassl.

\section{PLUMBAGINACEAE}

Plumbago scandens L.

POACEAE

Acroceras zizanioides (Kunth) Dandy

Axonopus cf. fissifolius (Raddi) Kuhlm.

Echinochloa colona (L.) Link

Echinochloa crus-galli (L.) P. Beauv.

Eleusine indica L.

Eriochloa punctata (L.) Desv. ex Ham.

Guadua sp.

Ichnanthus cf. nemoralis (Schrad. ex Schult.) Hitchc. \& Chase

Ichnanthus cf. procurrens (Nees ex Triana) Swallen

Lasiacis sorghoidea (Desv. ex Ham.) Hitchc. \& Chase

Megathyrsus maximus (Jacq.) B.K.Simon \& S.W.L.Jacobs

Olyra ciliatifolia Raddi

Olyra latifolia L.

Oplismenus hirtellus (L.) P. Beauv.

Panicum millegrana Pior.

Panicum pilosum $\mathrm{Sw}$.

Panicum trichanthum Nees

Pennisetum pedicellatum Trin.

Pereilema beyrichianum (Kunth) Hitch

Pharus lappulaceus Aubl.
Hábito CA1 CE2 MA3 AM4 CA4 CE4 MA4 PP4 PT4

\begin{tabular}{|c|c|c|c|c|c|c|c|c|c|c|}
\hline 1530 & erva & $\mathrm{x}$ & $\mathrm{x}$ & $\mathrm{x}$ & & $\mathrm{x}$ & $\mathrm{x}$ & $\mathrm{x}$ & & \\
\hline 259 & arb & $\mathrm{x}$ & $x$ & $\mathrm{x}$ & $\mathrm{x}$ & $x$ & $\mathrm{x}$ & $\mathrm{x}$ & & \\
\hline 364 & arb & & & $\mathrm{x}$ & & & & $\mathrm{x}$ & & \\
\hline 1013 & arb & & & & & & $\mathrm{x}$ & $\mathrm{x}$ & & \\
\hline 1180 & arb & & & & & & & & & \\
\hline 986 & arb & & & & & & & & & \\
\hline 280 & arb & $\mathrm{x}$ & $\mathrm{x}$ & $\mathrm{x}$ & $\mathrm{x}$ & & $\mathrm{x}$ & $\mathrm{x}$ & & \\
\hline 817 & erva & & & $\mathrm{x}$ & $\mathrm{x}$ & $\mathrm{x}$ & $\mathrm{x}$ & $\mathrm{x}$ & & \\
\hline 92 & trep & $\mathrm{x}$ & & $\mathrm{x}$ & $\mathrm{x}$ & $\mathrm{x}$ & & $\mathrm{x}$ & & \\
\hline 1174 & erva & $\mathrm{x}$ & $\mathrm{x}$ & $\mathrm{x}$ & $\mathrm{x}$ & $\mathrm{x}$ & $\mathrm{x}$ & $\mathrm{x}$ & & \\
\hline 1177 & erva & $\mathrm{x}$ & $\mathrm{x}$ & $\mathrm{x}$ & $\mathrm{x}$ & $\mathrm{x}$ & $\mathrm{x}$ & $\mathrm{x}$ & & \\
\hline 24 & erva & $\mathrm{x}$ & $\mathrm{x}$ & $\mathrm{x}$ & $\mathrm{x}$ & $\mathrm{x}$ & $\mathrm{x}$ & $\mathrm{x}$ & & \\
\hline 1059 & erva & & $\mathrm{x}$ & $\mathrm{x}$ & $\mathrm{x}$ & $\mathrm{x}$ & $\mathrm{x}$ & $\mathrm{x}$ & $\mathrm{X}$ & $\mathrm{X}$ \\
\hline 441 & erva & $\mathrm{x}$ & & & $x$ & $\mathrm{x}$ & $\mathrm{x}$ & $\mathrm{x}$ & $\mathrm{x}$ & $\mathrm{x}$ \\
\hline 535 & erva & $\mathrm{x}$ & & $\mathrm{x}$ & $\mathrm{x}$ & $x$ & $\mathrm{x}$ & $\mathrm{x}$ & & \\
\hline 1328 & erva & & & & & & & & & \\
\hline 1157 & erva & $\mathrm{x}$ & $\mathrm{x}$ & $\mathrm{x}$ & $\mathrm{x}$ & $x$ & $\mathrm{x}$ & $\mathrm{x}$ & & \\
\hline 747 & erva & $\mathrm{x}$ & $\mathrm{x}$ & $\mathrm{x}$ & $x$ & $\mathrm{x}$ & $\mathrm{x}$ & $\mathrm{x}$ & & \\
\hline 525 & erva & $\mathrm{x}$ & $\mathrm{x}$ & $\mathrm{x}$ & $\mathrm{x}$ & $x$ & $\mathrm{x}$ & $\mathrm{x}$ & & \\
\hline 711 & erva & & $\mathrm{x}$ & & $\mathrm{x}$ & $\mathrm{x}$ & $\mathrm{x}$ & $\mathrm{x}$ & & $\mathrm{x}$ \\
\hline 357 & erva & $\mathrm{x}$ & $\mathrm{x}$ & $\mathrm{x}$ & $\mathrm{x}$ & $\mathrm{x}$ & $\mathrm{x}$ & $\mathrm{x}$ & & $\mathrm{x}$ \\
\hline 276 & erva & $\mathrm{x}$ & $\mathrm{x}$ & $\mathrm{x}$ & $\mathrm{x}$ & $\mathrm{x}$ & $\mathrm{x}$ & $\mathrm{x}$ & & \\
\hline 511 & erva & $\mathrm{x}$ & $\mathrm{x}$ & $\mathrm{x}$ & $x$ & $\mathrm{x}$ & $\mathrm{x}$ & $\mathrm{x}$ & & \\
\hline 1175 & erva & $\mathrm{x}$ & $\mathrm{x}$ & $\mathrm{x}$ & $\mathrm{x}$ & $\mathrm{x}$ & $\mathrm{x}$ & $\mathrm{x}$ & $\mathrm{x}$ & $\mathrm{x}$ \\
\hline 356 & erva & $\mathrm{x}$ & $\mathrm{x}$ & $\mathrm{x}$ & $\mathrm{x}$ & $\mathrm{x}$ & $\mathrm{x}$ & $\mathrm{x}$ & $\mathrm{x}$ & $\mathrm{x}$ \\
\hline 617 & erva & & $\mathrm{x}$ & $\mathrm{x}$ & $\mathrm{x}$ & $\mathrm{x}$ & $\mathrm{x}$ & $\mathrm{x}$ & $\mathrm{x}$ & \\
\hline 536 & erva & $\mathrm{x}$ & $\mathrm{x}$ & & $\mathrm{x}$ & $\mathrm{x}$ & $\mathrm{x}$ & & & \\
\hline 33 & erva & & & $\mathrm{x}$ & & & $\mathrm{x}$ & & & \\
\hline 358 & erva & $\mathrm{x}$ & $\mathrm{x}$ & $\mathrm{x}$ & $\mathrm{X}$ & $x$ & $\mathrm{x}$ & $\mathrm{x}$ & & \\
\hline
\end{tabular}




\section{FAMÍLIA/ESPÉCIE}

Rhipidocladum $\mathrm{sp}$.

Setaria poiretiana (Schult.) Kunth

Setaria vulpiseta (Lam.) Roem. \& Schult.

POLYGONACEAE

Coccoloba sp.

Polygonum sp.

Triplaris sp.

PORTULACACEAE

Talinum paniculatum (Jacq.) Gaertn.

RHAMNACEAE

Gouania polygama (Jacq.) Urb.

Reissekia cf. smilacina (Sm.) Steud.

ROSACEAE

Rubus sp.

RUBIACEAE

Chomelia pohliana Müll.Arg.

Chomelia sp.

Coffea arabica $\mathrm{L}$.

Cordiera sessilis (Vell.) Kuntze

Coutarea hexandra (Jacq.) K. Schum.

Galianthe hispidula (A. Rich. ex DC.) E.L. Cabral \& Bacigalupo

Hamelia patens Jacq.

Psychotria carthagenensis Jacq.

Psychotria cf. remota Benth.

Psychotria minutiflora Müll.Arg.

Psychotria subtriflora Müll.Arg.

Randia armata (Sw.) DC.

Richardia scabra L.

Rudgea recurva Müll.Arg.

RUTACEAE

Conchocarpus cf. pentandrus (Engl.) Kallunki \& Pirani

Galipea jasminiflora (A.St.-Hil.) Engl.

Metrodorea nigra A.St.-Hil.

Pilocarpus cf. pauciflorus A.St.-Hil.
Melo, P.H.A

Hábito CA1 CE2 MA3 AM4 CA4 CE4 MA4 PP4 PT4

$\begin{array}{llllllllll}1156 & \text { erva } & & & & & & & \\ 517 & \text { erva } & \text { x } & & & & & & \\ 359 & \text { erva } & \text { x } & \text { x } & \text { x } & \text { x } & \text { x } & \text { x } & \text { x }\end{array}$

2 arb

1079 erva

2481 árv

254 erva

$458 \quad$ trep

486 trep

$\mathrm{x}$

$1260 \quad$ arb

940 arb $\quad x \quad x$

1347 arb

$\begin{array}{lllllll}677 & \text { arb } & \text { x } & \text { x } & \text { x } & \text { x } & \text { x }\end{array}$

281 árv X

351 árv $\quad \mathrm{x} \quad \mathrm{x} \quad \mathrm{x} \quad \mathrm{x} \quad \mathrm{x} \quad \mathrm{x}$

365 arb $\quad$ x

$\begin{array}{lllllllllll}282 & \operatorname{arb} & \mathrm{x} & \mathrm{x} & \mathrm{x} & \mathrm{x} & \mathrm{x} & \mathrm{x} & \mathrm{x} & & \mathrm{x}\end{array}$

$\begin{array}{lllllllll}231 & \operatorname{arb} & \mathrm{x} & \mathrm{x} & \mathrm{x} & \mathrm{x} & \mathrm{x} & \mathrm{x} & \mathrm{x}\end{array}$

665 arb $\quad x$

796 arb $\quad$ x $\quad$ x $\quad$ x $\quad$ x

$\begin{array}{lllllllllll}277 & \text { arb } & \mathrm{x} & \mathrm{x} & \mathrm{x} & \mathrm{x} & \mathrm{x} & \mathrm{x}\end{array}$

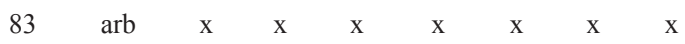

$\begin{array}{lllllllll}609 & \text { erva } & \mathrm{x} & \mathrm{x} & \mathrm{x} & \mathrm{x} & \mathrm{x} & \mathrm{x} & \mathrm{x}\end{array}$

287 arb $\quad x-x$

\begin{tabular}{|c|c|c|c|c|c|c|}
\hline $57 \quad$ arb & $\mathrm{x}$ & $\mathrm{x}$ & & & $\mathrm{x}$ & $\mathrm{x}$ \\
\hline árv & $\mathrm{x}$ & $\mathrm{x}$ & & & $\mathrm{x}$ & $\mathrm{x}$ \\
\hline 1009 & $\mathrm{x}$ & $\mathrm{x}$ & $\mathrm{x}$ & $\mathrm{x}$ & $\mathrm{x}$ & $\mathrm{x}$ \\
\hline arb & & $x$ & & & & $\mathrm{x}$ \\
\hline
\end{tabular}




\section{FAMÍLIA/ESPÉCIE}

Zanthoxylum monogynum A.St.-Hil.

\section{SALICACEAE}

Casearia gossypiosperma Briq.

Casearia lasiophylla Eichler

Casearia sylvestris Sw.

Prockia crucis P.Browne ex L.

Xylosma sp.

\section{SANTALACEAE}

Phoradendron piperoides (Kunth) Trel.

SAPINDACEAE

Allophylus racemosus Sw.

Cardiospermum sp.

Cupania vernalis Cambess.

Matayba guianensis Aubl.

Paullinia sp.

Serjania $\mathrm{sp}$

Urvillea sp.

SAPOTACEAE

Chrysophyllum marginatum (Hook. \& Arn.) Radlk.

Chrysophyllum viride Mart. \& Eichler

Pouteria grandiflora (A.DC.) Baehni

Pouteria sp.

\section{SCROPHULARIACEAE}

Buddleia cf. brasiliensis Jacq.

\section{SOLANACEAE}

Capsicum baccatum L.

Cestrum sp.1

Cestrum sp.2

Cestrum strigilatum Ruiz \& Pav.

Dyssochroma viridiflora (Sims) Miers.

Lycianthes repens Bitter

Physalis angulata L.

Solandra grandiflora Sw.

Solanum alternatopinnatum Steud.

Solanum americanum Mill.
Melo, P.H.A

Hábito CA1 CE2 MA3 AM4 CA4 CE4 MA4 PP4 PT4

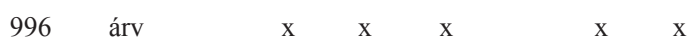

1238 árv

2434 árv

768 árv

225 árv

907 árv

238 erva

244 árv x $\quad$ x

827 trep

571 árv

2431 árv

246 trep

781 trep

315 trep

$\mathrm{X}$

1426

1417 árv

828 erva $\mathrm{x}$

362 arb

1682 arb

$672 \quad$ arb

855 arb $\mathrm{x} \quad \mathrm{x}$

1218 erva x

$\begin{array}{lllllllllllll}267 & \text { arb } & \mathrm{x} & \mathrm{x} & \mathrm{x} & \mathrm{x} & \mathrm{x} & \mathrm{x} & \mathrm{x} & \mathrm{x}\end{array}$

1411 trep $\quad \mathrm{x} \quad \mathrm{x} \quad \mathrm{x}$

549 trep $\quad$ x $\quad$ x $\quad$ x $\quad$ x

$\begin{array}{llllllllllllll}506 & \operatorname{arb} & \mathrm{x} & \mathrm{x} & \mathrm{x} & \mathrm{x} & \mathrm{x} & \mathrm{x} & \mathrm{x} & \mathrm{x} & \mathrm{x}\end{array}$ 
FAMÍLIA/ESPÉCIE

Solanum oocarpum Sendtn.

Solanum paniculatum L.

Solanum scuticum M.Nee

\section{TROPAEOLACEAE}

Tropaeolum cf. warmingianum Rohrb.

TYPHACEAE

Typha sp.

URTICACEAE

Cecropia glaziovii Snethl.

Cecropia pachystachya Trécul

Hemistylus brasiliensis Wedd. ex Warm.

Pilea hyalina Fenzl

Pilea microphylla (L.) Liebm.

Pilea sp.

Urera baccifera (L.) Gaudich. ex Wedd.

Urera cf. aurantiaca Wedd.

Urera cf. nitida (Vellozo) P.Brack

VERBENACEAE

Aloysia virgata (Ruiz \& Pav.) A.Juss.

Lantana camara L.

Lantana cf. fucata Lindl.

Lantana trifoliaL.

Petrea volubilis L.

VIOLACEAE

Hybanthus bigibbosus (A. St.-Hil.) Hassl.

VITACEAE

Cissus erosa subsp. erosa Rich.

Cissus serroniana (Glaz.) Lombardi

Cissus simsiana Schult. \& Schult.f.

Cissus sulcicaulis (Baker) Planch.

Cissus tinctoria Mart.

Cissus verticillata subsp. verticillata (L.) Nicolson \& C.E.Jarvis
Hábito CA1 CE2 MA3 AM4 CA4 CE4 MA4 PP4 PT4

$\begin{array}{llllllll}875 & \text { arb } & \text { x } & \text { x } & & & \text { x } & \text { x } \\ 306 & \text { arb } & \text { x } & \text { x } & \text { x } & \text { x } & \text { x } & \text { x } \\ 230 & \text { arb } & \text { x } & & \text { x } & & \text { x } & \text { x }\end{array}$

1019 trep

958 erva

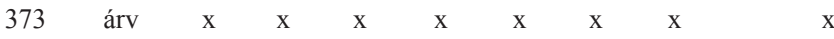

$540 \quad \operatorname{arb} \quad \mathrm{x} \quad \mathrm{x}$

1868 erva

387 erva $\quad \mathrm{x} \quad \mathrm{x} \quad \mathrm{x} \quad \mathrm{x} \quad \mathrm{x}$

1158 erva

$291 \quad \operatorname{arb} \quad \mathrm{x} \quad \mathrm{x} \quad \mathrm{x} \quad \mathrm{x} \quad \mathrm{x} \quad \mathrm{x}$

466 arb $x$

21 arb $\quad$ x $x$

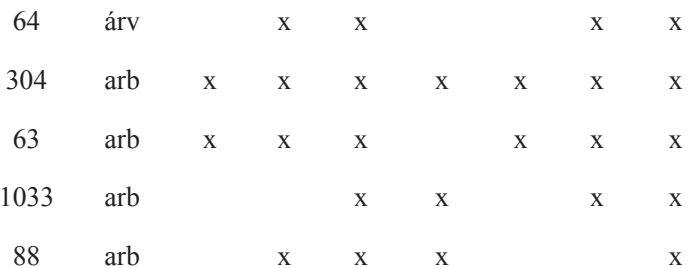

90 arb $\quad$ x $\quad$ x $\quad$ x

$\begin{array}{lllllllll}951 & \text { trep } & \mathrm{x} & \mathrm{x} & \mathrm{x} & \mathrm{x} & \mathrm{x} & \mathrm{x} & \mathrm{x} \\ 317 & \operatorname{trep} & & \mathrm{x} & \mathrm{x} & & & \mathrm{x} & \mathrm{x} \\ 1081 & \operatorname{trep} & \mathrm{x} & \mathrm{x} & \mathrm{x} & \mathrm{x} & \mathrm{x} & \mathrm{x} & \mathrm{x} \\ 331 & \operatorname{trep} & \mathrm{x} & \mathrm{x} & \mathrm{x} & \mathrm{x} & \mathrm{x} & \mathrm{x} & \mathrm{x} \\ 1220 & \operatorname{trep} & \mathrm{x} & & \mathrm{x} & \mathrm{x} & \mathrm{x} & \mathrm{x} & \mathrm{x} \\ & & & & & & & & \end{array}$

